\title{
Looking for the left sneutrino LSP with displaced-vertex searches
}

\author{
Iñaki Lara, ${ }^{1,2^{*}}$ Daniel E. López-Fogliani, ${ }^{3,4 \dagger}$ Carlos Muñoz, ${ }^{1,2 \ddagger}$ Natsumi Nagata, ${ }^{5 \S}$ \\ Hidetoshi Otono, ${ }^{6}$ and Roberto Ruiz de Austri ${ }^{7 \pi}$ \\ ${ }^{1}$ Departamento de Física Teórica, Universidad Autónoma de Madrid (UAM), \\ Campus de Cantoblanco, 28049 Madrid, Spain \\ ${ }^{2}$ Instituto de Física Teórica UAM-CSIC, Campus de Cantoblanco, 28049 Madrid, Spain \\ ${ }^{3}$ Instituto de Física de Buenos Aires UBA \& CONICET, Departamento de Física, Facultad de Ciencia \\ Exactas y Naturales, Universidad de Buenos Aires, 1428 Buenos Aires, Argentina \\ ${ }^{4}$ Pontificia Universidad Católica Argentina, 1107 Buenos Aires, Argentina \\ ${ }^{5}$ Department of Physics, University of Tokyo, Tokyo 113-0033, Japan \\ ${ }^{6}$ Research Center for Advanced Particle Physics, Kyushu University, Fukuoka 819-0395, Japan \\ ${ }^{7}$ Instituto de Física Corpuscular CSIC-UV, c/Catedrático José Beltrán 2, 46980 Paterna, Valencia, Spain
}

(Received 13 April 2018; published 2 October 2018)

\begin{abstract}
We analyze a displaced dilepton signal expected at the LHC for a tau left sneutrino as the lightest supersymmetric particle with a mass in the range $45-100 \mathrm{GeV}$. The sneutrinos are pair produced via a virtual $W, Z$ or $\gamma$ in the $s$ channel and, given the large value of the tau Yukawa coupling, their decays into two dileptons or a dilepton plus missing transverse energy from neutrinos can be significant. The discussion is carried out in the framework of the $\mu \nu \mathrm{SSM}$, where the presence of $R$-parity violating couplings involving right-handed neutrinos solves the $\mu$ problem and can reproduce the neutrino data. To probe the tau left sneutrinos we compare the predictions of this scenario with the ATLAS search for longlived particles using displaced lepton pairs in $p p$ collisions at $\sqrt{s}=8 \mathrm{TeV}$, allowing us to constrain the parameter space of the model. We also consider an optimization of the trigger requirements used in existing displaced-vertex searches by means of a high level trigger that exploits tracker information. This optimization is generically useful for a light metastable particle decaying into soft charged leptons. The constraints on the sneutrino turn out to be more stringent. We finally discuss the prospects for the $13 \mathrm{TeV}$ LHC searches as well as further potential optimizations.
\end{abstract}

DOI: 10.1103/PhysRevD.98.075004

\section{INTRODUCTION}

Clarifying whether or not low-energy supersymmetry (SUSY) exists in nature is one of the main goals of the LHC. If $R$-parity $\left(R_{p}\right)$ is not conserved, the lightest supersymmetric particle (LSP) decays into standard model particles and displaced vertices can appear, implying that dedicated analyses to detect SUSY are necessary. The " $\mu$ from $\nu$ " supersymmetric standard model $(\mu \nu \mathrm{SSM})[1,2]$ is a SUSY framework where the $\mu$ problem is solved and the

\footnotetext{
*inaki.lara@csic.es

†aniel.lopez@df.uba.ar

"c.munoz@uam.es

\$natsumi@hep-th.phys.s.u-tokyo.ac.jp

"otono@phys.kyushu-u.ac.jp

"rruiz@ific.uv.es
}

Published by the American Physical Society under the terms of the Creative Commons Attribution 4.0 International license. Further distribution of this work must maintain attribution to the author(s) and the published article's title, journal citation, and DOI. Funded by SCOAP ${ }^{3}$. neutrino data can be reproduced [1-6]. For this mechanism to work, the presence of trilinear couplings involving righthanded neutrino superfields is crucial. As a consequence, $R_{p}$ is explicitly broken $\left(R_{p}\right)$. Given the $R_{p}$ in the $\mu \nu \mathrm{SSM}$, essentially all SUSY particles are potential candidates for LSPs, and therefore dedicated analyses of the LHC phenomenology associated to each candidate are crucial to test SUSY. As we will discuss below, in this work we will focus our analysis on the left sneutrino as the LSP candidate.

The simplest superpotential of the $\mu \nu \mathrm{SSM}[1,2]$ is built with one right-handed neutrino superfield $\hat{\nu}^{c}$ :

$$
\begin{aligned}
W= & \epsilon_{a b}\left(Y_{e_{i j}} \hat{H}_{d}^{a} \hat{L}_{i}^{b} \hat{e}_{j}^{c}+Y_{d_{i j}} \hat{H}_{d}^{a} \hat{Q}_{i}^{b} \hat{d}_{j}^{c}+Y_{u_{i j}} \hat{H}_{u}^{b} \hat{Q}^{a} \hat{u}_{j}^{c}\right) \\
& +\epsilon_{a b}\left(Y_{\nu_{i}} \hat{H}_{u}^{b} \hat{L}_{i}^{a} \hat{\nu}^{c}-\lambda \hat{\nu}^{c} \hat{H}_{u}^{b} \hat{H}_{d}^{a}\right)+\frac{1}{3} \kappa \hat{\nu}^{c} \hat{\nu}^{c} \hat{\nu}^{c}
\end{aligned}
$$

where the summation convention is implied on repeated indexes, with $a, b=1,2 S U(2)_{L}$ indices and $i, j, k=1,2,3$ the usual family indices of the standard model. In this equation we have neglected the conventional trilinear $\mathbb{R}_{p}$ 
couplings (see e.g., the discussion in Ref. [7]). The terms in the second line are characteristic of the $\mu \nu \mathrm{SSM}$. The first one contains the Dirac Yukawa couplings for neutrinos. The last two, after the electroweak symmetry breaking induced by the soft SUSY-breaking terms, generate dynamically the $\mu$ term, $\mu=\lambda \frac{v_{R}}{\sqrt{2}}$, and the Majorana mass for the right-handed neutrino $\nu_{R}, m_{\mathcal{M}}=2 \kappa \frac{v_{R}}{\sqrt{2}}$, respectively. With the choice of $C P$ conservation, we have defined the vacuum expectation value (VEV) of the right sneutrino $\tilde{\nu}_{R}$, as $\left\langle\tilde{\nu}_{R}\right\rangle=\frac{v_{R}}{\sqrt{2}}$. SUSY particles do not appear in pairs in these terms, thus they generate $\mathbb{R}_{p}$ couplings harmless with respect to proton decay. In the limit $Y_{\nu_{i}} \rightarrow 0, \hat{\nu}^{c}$ can be identified in the superpotential as a pure singlet superfield without lepton number, and $R_{p}$ is not broken. Thus, the neutrino Yukawa couplings $Y_{\nu_{i}}$ are the parameters which determine the $\mathbb{R}_{p}$ in the $\mu \nu \mathrm{SSM}$, and as a consequence this violation is small.

In addition to the VEV for the $\tilde{\nu}_{R}$, the minimization of the scalar potential induces VEVs for the neutral Higgses $H_{d}^{0}$ and $H_{u}^{0},\left\langle H_{d, u}^{0}\right\rangle=\frac{v_{d, u}}{\sqrt{2}}$, and the left sneutrinos $\tilde{\nu}_{i}$, $\left\langle\tilde{\nu}_{i}\right\rangle=\frac{v_{i}}{\sqrt{2}}$, with $v_{i} \sim Y_{\nu_{i}} v_{u}$. The new couplings and sneutrino VEVs in the $\mu \nu \mathrm{SSM}$ induce new mixing of states. The associated mass matrices were studied in detail in Refs. [2,4,8]. Summarizing, in the case of one right-handed neutrino superfield, there are eight neutral fermions (neutralinos-neutrinos), five charged fermions (charginosleptons), seven charged scalars (charged Higgses-sleptons), and six neutral scalars and five neutral pseudoscalars (Higgses-sneutrinos). It is worth noticing here that whereas $v_{R} \sim \mathrm{TeV}, v_{i} \lesssim 10^{-4} \mathrm{GeV}$ because of the small contributions $Y_{\nu_{i}} \lesssim 10^{-6}$ whose size is determined by the electroweak-scale seesaw of the $\mu \nu \mathrm{SSM}[1,2]$. In fact, this is a generalized seesaw since the left-handed neutrinos mix not only with the right-handed one, but also with the neutralinos. Given that the mass matrix is of rank 6, only one of the light neutrinos gets a nonvanishing tree-level contribution to its mass, whereas the other two get their masses at one loop. The tree-level mass is given by

$$
m_{\nu}=\frac{1}{4 M_{\mathrm{eff}}} \sum_{i}\left[v_{i}^{2}+v_{d}\left(\frac{2 v_{i} Y_{\nu_{i}}}{\lambda}+\frac{v_{d} Y_{\nu_{i}}^{2}}{\lambda^{2}}\right)\right],
$$

with

$M_{\text {eff }} \equiv M\left[1-\frac{v^{2}}{\sqrt{2} M\left(\kappa v_{R}^{2}+\lambda v_{d} v_{u}\right) \lambda v_{R}}\left(\kappa v_{R}^{2} \frac{v_{d} v_{u}}{v^{2}}+\frac{1}{4} \lambda v^{2}\right)\right]$,

where $v^{2} \equiv v_{d}^{2}+v_{u}^{2}$, and

$$
\frac{1}{M} \equiv \frac{g^{2}}{M_{1}}+\frac{g^{2}}{M_{2}}
$$

Notice that we can simplify Eq. (1.2) taking into account that the second term is proportional to $v_{d}$, and therefore considering it negligible in the limit of large or even moderate $\tan \beta \equiv v_{u} / v_{d}$ provided that $\lambda$ is not too small. In Eq. (1.3), the second term is also negligible in this limit, and for typical values of the parameters involved in the seesaw also the third one, i.e., $M_{\text {eff }} \approx M$. Under these assumptions, the first term in Eq. (1.2) is generated only through the mixing of left-handed neutrinos with gauginos, and we arrive to the approximate formula $m_{\nu} \approx \sum_{i} v_{i}^{2} / 4 M$.

A promptly decaying sneutrino as the LSP was analyzed in Ref. [8], with a decay length $\lesssim 0.1 \mathrm{~mm}$. In this work, we will analyze the interesting case of displaced vertices of the order of the millimeter, when a sneutrino in the range of masses $45-100 \mathrm{GeV}$ is allowed. The lower bound imposed not to disturb the decay width of the $Z$. We will focus on the simplest case of the $\mu \nu \mathrm{SSM}$ with one right-handed neutrino superfield discussed above, and leave the case of three families where all the neutrinos get contributions to their masses at tree level for a forthcoming publication [9].

In the $\mu \nu \mathrm{SSM}$, the mixing between left sneutrinos and Higgses/right sneutrino is small, and this implies that the left sneutrino dominates the LSP composition. Besides, scalar and pseudoscalar states, which in our convention are defined as $\tilde{\nu}_{i}=\frac{1}{\sqrt{2}}\left(\tilde{\nu}_{i}^{\mathcal{R}}+i \tilde{\nu}_{i}^{\mathcal{I}}\right)$, are co-LSPs since they have essentially degenerate masses, $m_{\tilde{\nu}_{i}^{\mathcal{R}}} \approx m_{\tilde{\nu}_{i}^{I}} \equiv m_{\tilde{\nu}_{i}}$. Their approximate tree-level value is

$$
m_{\tilde{\nu}_{i}}^{2} \approx \frac{Y_{\nu_{i}} v_{u}}{2 v_{i}} v_{R}\left(-\sqrt{2} A_{\nu_{i}}-\kappa v_{R}+\frac{\lambda v_{R}}{\tan \beta}\right)
$$

where $A_{\nu_{i}}$ are the trilinear parameters in the soft Lagrangian, $-\epsilon_{a b} T_{\nu_{i}} H_{u}^{b} \tilde{L}_{i L}^{a} \tilde{\nu}_{R}^{*}$, defining $T_{\nu_{i}} \equiv A_{\nu_{i}} Y_{\nu_{i}}$ and with the summation convention not applied in this case. Taking all this into account, scalar and pseudoscalar sneutrinos are dominantly pair produced via a Drell-Yan process mediated by a virtual $W, Z$ or $\gamma$. Besides, the case of the tau left sneutrino $\left(\tilde{\nu}_{\tau}\right)$ LSP turns out to be particularly interesting, because of the large value of the tau Yukawa coupling which can give rise to significant branching ratios (BRs) for decays to ${ }^{1} \tau \tau$ and $\tau \ell$.

To probe the $\tilde{\nu}_{\tau}$ LSP, the dilepton displaced-vertex searches are found to be most promising. We compare the predictions of our scenario with the ATLAS search [10] for long-lived particles using displaced lepton pairs $\ell \ell$ in $p p$ collisions at $\sqrt{s}=8 \mathrm{TeV}$, which allows us to constrain the parameter space of the model. Nevertheless, the existing searches $[10,11]$ are designed for a generic purpose and

\footnotetext{
${ }^{1}$ In what follows, the symbol $\ell$ will be used for an electron or a muon, $\ell=e, \mu$, and charge conjugation of fermions is to be understood where appropriate.
} 


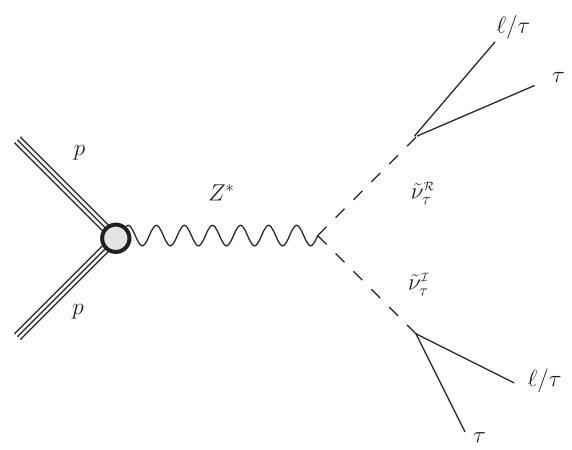

(a) $Z$ channel

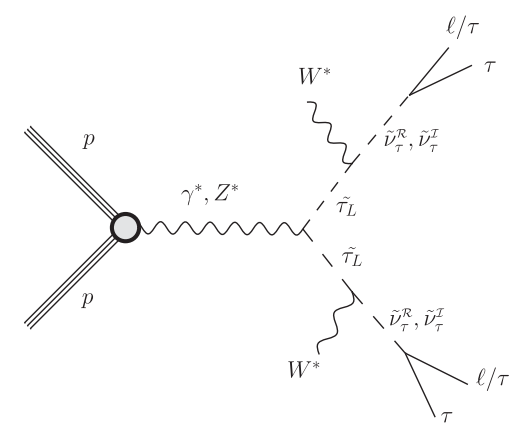

(b) $\gamma, Z$ channels

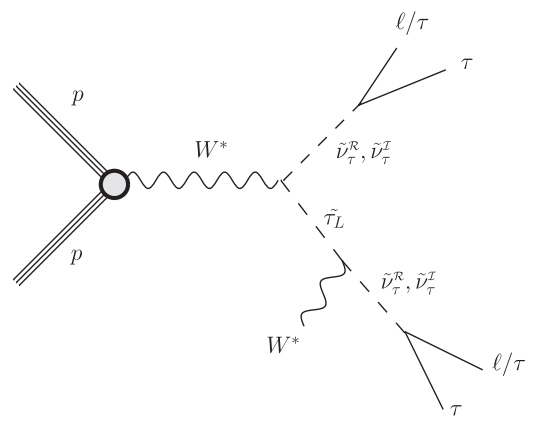

(c) $W$ channel

FIG. 1. Decay channels into two $\tau \ell / \tau$, from a pair production at the LHC of scalar and pseudoscalar tau left sneutrinos co-LSPs. Decay channels into one $\tau \ell / \tau$ plus neutrinos are the same but substituting in (a), (b) and (c) one of the two vertices by a twoneutrino vertex.

thus not optimized for light metastable particles such as the $\tilde{\nu}_{\tau}$. We therefore consider also a possibility of improving these searches by lowering trigger thresholds, relying on a high level trigger that utilizes tracker information. As it turns out, this optimization is quite feasible and considerably improves the sensitivity of the displaced-vertex searches. We also consider an optimization of the $13 \mathrm{TeV}$ LHC searches and show the prospects for investigating the parameter space of our scenario by searching for the $\tilde{\nu}_{\tau}$ at the $13 \mathrm{TeV}$ LHC run. Possibilities of further improvements for these searches will also be discussed.

The paper is organized as follows. In Sec. II, we will introduce the phenomenology of the $\tilde{\nu}_{\tau}$ LSP, studying its pair production channels at the LHC, as well as the signals. These consist of two dileptons or a dilepton plus missing transverse energy (MET) from the sneutrino decays. On the way, we will analyze the decay widths, BRs and decay lengths. In Sec. III, we consider first the existing dilepton displaced-vertex searches, and discuss its feasibility and significance on $\tilde{\nu}_{\tau}$ searches. Then, we study an optimization by using a high level trigger with tracker information. We also show our prescription for recasting the ATLAS 8-TeV result [10] to the case of the $\tilde{\nu}_{\tau}$. We then show the current reach of this search on the parameter space of our scenario based on the ATLAS 8-TeV result [10], and the prospects for the 13-TeV searches in Sec. IV. Our conclusions and prospects for future work are presented in Sec. V.

\section{LEFT SNEUTRINO LSP PHENOMENOLOGY IN THE $\mu \nu$ SSM}

The dominant pair production channels of sleptons at large hadron colliders were studied in Refs. [12-17]. In Fig. 1, we can see the production channels at the LHC for the case of the $\tilde{\nu}_{\tau}$ LSP which is the one interesting for our analysis. The direct production of Fig. 1(a) occurs via a $Z$ channel giving rise to a pair of scalar and pseudoscalar left sneutrinos. As discussed in the Introduction, these states have essentially degenerate masses and are therefore co-LSPs. On the other hand, since the left stau is in the same $S U(2)$ doublet as the tau left sneutrino, it becomes the next-to-LSP (NLSP). The mass splitting is mainly due to the usual D-term contribution. Similar to the MSSM, one obtains that the difference of the squared masses is given by $-m_{W}^{2} \cos 2 \beta$. Taking into account the particular values of $\tan \beta$ and the sneutrino mass (as well as the loop corrections to sneutrino and stau masses), the typical mass difference is of about $20-30 \mathrm{GeV}$. Thus the direct production and decay of the left stau is another important source of the $\tilde{\nu}_{\tau}$ LSP. In particular, pair production can be obtained through a $\gamma$ or $Z$ decaying into two staus, as shown in Fig. 1(b), with the latter dominantly decaying into a (scalar or pseudoscalar) sneutrino plus an off-shell $W$ producing a soft meson or a pair of a charged lepton and a neutrino. Besides, sneutrinos can be pair produced through a $W$ decaying into a stau and 
TABLE I. Number of sneutrino pairs produced through the different channels shown in Fig. 1 at 8 and 13 TeV with integrated luminosity of 20.3 and $300 \mathrm{fb}^{-1}$, respectively.

\begin{tabular}{|c|c|c|c|c|c|c|c|c|}
\hline \multirow[b]{2}{*}{$m_{\tilde{\nu}_{\tau}}(\mathrm{GeV})$} & \multicolumn{4}{|c|}{$8 \mathrm{TeV}$} & \multicolumn{4}{|c|}{$13 \mathrm{TeV}$} \\
\hline & 50 & 60 & 80 & 100 & 50 & 60 & 80 & 100 \\
\hline Through $Z$ boson & 66,400 & 22,370 & 6,090 & 2,448 & $1,794,000$ & 623,000 & 179,800 & 75,700 \\
\hline Through $W$ boson & 66,600 & 34,870 & 12,840 & 5,915 & $1,860,000$ & 997,000 & 385,600 & 184,800 \\
\hline Through $\gamma, Z$ bosons & 6,585 & 3,954 & 1,703 & 851 & 226,300 & 139,400 & 62,550 & 32,683 \\
\hline
\end{tabular}

a (scalar or pseudoscalar) sneutrino as shown in Fig. 1(c), with the stau decaying as before. The number of sneutrino pairs produced through these channels at 8 and $13 \mathrm{TeV}$ for integrated luminosities of 20.3 and $300 \mathrm{fb}^{-1}$, respectively, are shown in Table I.

In Fig. 1, we also show the detectable decay of the pairproduced $\tilde{\nu}_{\tau}$ into $\tau \ell / \tau$. As a result of the mixing between left sneutrinos and Higgses, the sizable decay of $\tilde{\nu}_{\tau}$ into $\tau \tau$ is possible because of the large value of the tau Yukawa coupling. Other sizable decays into $\tau \ell / \tau$ can occur through the Yukawa interaction of $\tilde{\nu}_{\tau}$ with $\tau$ and charged Higgsinos, via the mixing between the latter and $\ell$ or $\tau$. To analyze these processes we can write approximate formulas for the partial decay widths of the scalar/pseudoscalar tau left sneutrino. The one into $\tau \tau$ is given by

$$
\Gamma\left(\tilde{\nu}_{\tau} \rightarrow \tau \tau\right) \approx \frac{m_{\tilde{\nu}_{\tau}}}{16 \pi}\left(Y_{\tau} Z_{\tilde{\nu}_{\tau} H_{d}}^{H / A}-Y_{\nu_{\tau}} \frac{Y_{\tau}}{\lambda}\right)^{2}
$$

where $Y_{\tau} \equiv Y_{e_{33}}$, and $Z^{H / A}$ is the matrix which diagonalizes the mass matrix for the scalar/pseudoscalar Higgses $\left(H_{d}, H_{u}, \tilde{\nu}_{R}, \tilde{\nu}_{i}\right)[2]$. The latter is determined by the neutrino Yukawas, which are the order parameters of the $R_{p}$. The contribution of $\lambda$ in the second term of Eq. (2.1) is due to the charged Higgsino mass that can be approximated by the value of $\mu$. The partial decay width into $\tau \ell$ can then be approximated for both sneutrino states by the second term of Eq. (2.1) with the substitution $Y_{\nu_{\tau}} \rightarrow Y_{\nu_{\ell}}$ :

$$
\Gamma\left(\tilde{\nu}_{\tau} \rightarrow \tau \ell\right) \approx \frac{m_{\tilde{\nu}_{\tau}}}{16 \pi}\left(Y_{\nu_{\ell}} \frac{Y_{\tau}}{\lambda}\right)^{2}
$$

On the other hand, the gauge interactions of $\tilde{\nu}_{\tau}$ with neutrinos and Binos (Winos) can produce also a large decay width into neutrinos, via the gauge mixing between these gauginos and neutrinos. This partial decay width can be approximated for scalar and pseudoscalar sneutrinos as

$$
\sum_{i} \Gamma\left(\tilde{\nu}_{\tau} \rightarrow \nu_{\tau} \nu_{i}\right) \approx \frac{m_{\tilde{\nu}_{\tau}}}{16 \pi} \frac{1}{2 M^{2}} \sum_{i} v_{i}^{2}
$$

with $M$ a kind of average of Bino and Wino masses defined in Eq. (1.4). The relevant diagrams for $\tilde{\nu}_{\tau}$ searches that include this decay mode are the same as in Fig. 1, but substituting one of the $\tau \ell / \tau$ vertices by a two-neutrino vertex.

Let us remark that other decay channels of the $\tilde{\nu}_{\tau}$ can be present and have been taken into account in our numerical computation, but they turn out to be negligible for the sneutrino masses that we are interested in this work. For example, decay to bottoms can occur through a term similar to the first one of Eq. (2.1) with the substitution of $Y_{\tau}$ by $Y_{b}$. As we will comment below and was discussed in Ref. [8], this term is very small. In particular, it is negligible with respect to the second one in Eq. (2.1) which is present for decays into leptons.

It is also worth noticing here that because the $\tilde{\nu}_{\tau}$ in the $\mu \nu \mathrm{SSM}$ has several relevant decay modes, the LEP lower bound on the sneutrino mass of about $90 \mathrm{GeV}$ [18-23] obtained under the assumption of BR one to leptons, via trilinear $R_{p}$ couplings, is not directly applicable in this case. We have checked that no constraint on the $\tilde{\nu}_{\tau}$ mass is obtained from these searches in the cases studied in this work. We have obtained similar conclusions from LEP mono-photon search (gamma + MET) [24], and LHC mono-photon and mono-jet (jet + MET) searches $[25,26]$. Concerning LEP searches for staus [18-23], in the $\mu \nu \mathrm{SSM}$ the left stau does not decay directly but through an off-shell $W$ and a $\tilde{\nu}_{\tau}$. Thus, searches for its direct decay are not relevant in this model. On the other hand, the sneutrino mass can in principle be constrained using searches for final states as those produced in the $\mu \nu \mathrm{SSM}$. However, we have checked that this is not the case. For example, for the final state $\tau \mu \mu \nu \tau \mu \mu \nu$ [see Fig. 1(b)], taking into account the value of the production cross section at LEP for a pair of left staus, and the BRs of $W$ into $\mu \nu$ and $\tilde{\nu}_{\tau}$ into $\tau \mu$, no effective constraint is obtained in our scenario. For the other possible topologies, with $W$ into quarks or $\tilde{\nu}_{\tau}$ into $\nu \nu$, the results of the analyses turn out to be the same, as shown in Table II. It is straightforward to see there that our scenario is unconstrained, even considering the most disfavored (and unrealistic) values for the branching ratios, $\operatorname{BR}(\tilde{\nu} \rightarrow \ell \tau)=1$ or $\operatorname{BR}(\tilde{\nu} \rightarrow 2 \nu)=1$. In addition, we are not taking into account the possible effect of the different geometry of the decays when comparing with the final states considered in the searches, which are not originated in the same manner.

To analyze now the BRs into leptons, we have to focus on the decay channels where the $\tau$ 's in the final state decay leptonically. Let us study e.g., the BR to $\mu \mu$, since the $e e$ 
TABLE II. Possible topologies emerging from the production of a pair of tau left sneutrinos from staus. In the last two columns the production cross section of the most constrained signal, considering the worst-case scenario, is compared with the experimental upper limit. The values $\mathrm{BR}(W \rightarrow \mu \nu)=0.1063, \mathrm{BR}\left(W \rightarrow q q^{\prime}\right)=0.6741$, and $\mathrm{BR}(\tau \rightarrow \mu \nu)=0.1739$ are used for the computation, as well as $1 / 4$ as the maximum value of $\operatorname{BR}(\tilde{\nu} \rightarrow \ell \tau) \times \operatorname{BR}(\tilde{\nu} \rightarrow 2 \nu)$. In order to simplify the notation, $\operatorname{BR}(\tilde{\nu} \rightarrow \ell \tau)$ means the sum of the three BRs to $e \tau, \mu \tau$ and $\tau \tau$, and the factors $1 / 3$ are coming from considering the different channels.

\begin{tabular}{|c|c|c|c|}
\hline Process & Topology & Signal cross section upper limit & Exclusion \\
\hline $\begin{array}{l}\tilde{\tau}^{ \pm} \tilde{\tau}^{\mp} \rightarrow 2\left(W^{ \pm} \rightarrow \ell^{ \pm} \nu\right) \\
\quad+2(\tilde{\nu} \rightarrow \ell \tau)\end{array}$ & $4 \ell+2 \tau+E_{\mathrm{T}}^{\mathrm{miss}}$ & $\begin{array}{l}(0.13 \mathrm{pb}) \times(0.1063)^{2} \times \mathrm{BR}(\tilde{\nu} \rightarrow \ell \tau)^{2} \\
\quad \times\left(\frac{1}{3}\right)^{2} \leq 1.6 \times 10^{-4} \mathrm{pb}\end{array}$ & $\begin{array}{l}\text { Fig. } 14 \text { of Ref. [22] } \\
1.5 \times 10^{-2} \mathrm{pb}\end{array}$ \\
\hline $\begin{array}{l}\tilde{\tau}^{ \pm} \tilde{\tau}^{\mp} \rightarrow 2\left(W^{ \pm} \rightarrow \ell^{ \pm} \nu\right) \\
\quad+(\tilde{\nu} \rightarrow \ell \tau)+(\tilde{\nu} \rightarrow 2 \nu)\end{array}$ & $3 \ell+\tau+E_{\mathrm{T}}^{\text {miss }}$ & $\begin{array}{l}(0.13 \mathrm{pb}) \times(0.1063)^{2} \times 2 \\
\quad \times \mathrm{BR}(\tilde{\nu} \rightarrow \ell \tau) \times \frac{1}{3} \times(0.1739) \\
\quad \times \mathrm{BR}(\tilde{\nu} \rightarrow 2 \nu) \leq 4.3 \times 10^{-5} \mathrm{pb}\end{array}$ & $\begin{array}{l}\text { Fig. } 18 \text { of Ref. [22] } \\
2 \times 10^{-2} \mathrm{pb}\end{array}$ \\
\hline $\begin{array}{l}\tilde{\tau}^{ \pm} \tilde{\tau}^{\mp} \rightarrow 2\left(W^{ \pm} \rightarrow \ell^{ \pm} \nu\right) \\
\quad+2(\tilde{\nu} \rightarrow 2 \nu)\end{array}$ & $2 \ell+E_{\mathrm{T}}^{\mathrm{miss}}$ & $\begin{array}{l}(0.13 \mathrm{pb}) \times(0.1063)^{2} \times \mathrm{BR}(\tilde{\nu} \rightarrow 2 \nu)^{2} \\
\quad \leq 1.5 \times 10^{-3} \mathrm{pb}\end{array}$ & $\begin{array}{l}\text { Fig. } 6 \text { of Ref. [22] } \\
\quad 6 \times 10^{-2} \mathrm{pb}\end{array}$ \\
\hline $\begin{array}{l}\tilde{\tau}^{ \pm} \tilde{\tau}^{\mp} \rightarrow\left(W^{ \pm} \rightarrow \ell^{ \pm} \nu\right) \\
\quad+\left(W^{ \pm} \rightarrow q q^{\prime}\right)+2(\tilde{\nu} \rightarrow \ell \tau)\end{array}$ & $3 \ell+2 \tau+$ jets $+E_{\mathrm{T}}^{\text {miss }}$ & $\begin{array}{l}(0.13 \mathrm{pb}) \times 2 \times(0.1063) \times(0.6741) \\
\quad \times \mathrm{BR}(\tilde{\nu} \rightarrow \ell \tau)^{2} \times\left(\frac{1}{3}\right)^{2} \leq 2.1 \times 10^{-3} \mathrm{pb}\end{array}$ & $\begin{array}{l}\text { Fig. } 18 \text { of Ref. [22] } \\
2 \times 10^{-2} \mathrm{pb}\end{array}$ \\
\hline $\begin{array}{l}\tilde{\tau}^{ \pm} \tilde{\tau}^{\mp} \rightarrow\left(W^{ \pm} \rightarrow \ell^{ \pm} \nu\right) \\
\quad+\left(W^{ \pm} \rightarrow q q^{\prime}\right)+(\tilde{\nu} \rightarrow \ell \tau) \\
\quad+(\tilde{\nu} \rightarrow 2 \nu)\end{array}$ & $2 \ell+\tau+$ jets $+E_{\mathrm{T}}^{\text {miss }}$ & $\begin{array}{l}(0.13 \mathrm{pb}) \times 2 \times(0.1063) \times(0.6741) \\
\quad \times 2 \times \mathrm{BR}(\tilde{\nu} \rightarrow \ell \tau) \times\left(\frac{1}{3}\right) \times \mathrm{BR}(\tilde{\nu} \rightarrow 2 \nu) \\
\quad \leq 3.1 \times 10^{-3} \mathrm{pb}\end{array}$ & $\begin{array}{l}\text { Fig. } 12 \text { of Ref. [22] } \\
5 \times 10^{-2} \mathrm{pb}\end{array}$ \\
\hline $\begin{array}{l}\tilde{\tau}^{ \pm} \tilde{\tau}^{\mp} \rightarrow\left(W^{ \pm} \rightarrow \ell^{ \pm} \nu\right) \\
\quad+\left(W^{ \pm} \rightarrow q q^{\prime}\right)+2(\tilde{\nu} \rightarrow 2 \nu)\end{array}$ & $\ell+$ jets $+E_{\mathrm{T}}^{\text {miss }}$ & $\begin{array}{l}(0.13 \mathrm{pb}) \times 2 \times(0.1063) \times(0.6741) \\
\quad \times \mathrm{BR}(\tilde{\nu} \rightarrow 2 \nu)^{2} \leq 1.9 \times 10^{-2} \mathrm{pb}\end{array}$ & $\begin{array}{l}\text { Fig. } 6 \text { of Ref. [27] } \\
5 \times 10^{-2} \mathrm{pb}\end{array}$ \\
\hline $\begin{array}{l}\tilde{\tau}^{ \pm} \tilde{\tau}^{\mp} \rightarrow 2\left(W^{ \pm} \rightarrow q q^{\prime}\right) \\
\quad+2(\tilde{\nu} \rightarrow \ell \tau)\end{array}$ & $2 \ell+2 \tau+$ jets & $\begin{array}{l}(0.13 \mathrm{pb}) \times(0.6741)^{2} \times \mathrm{BR}(\tilde{\nu} \rightarrow \ell \tau)^{2} \\
\quad \times\left(\frac{1}{3}\right)^{2} \leq 6.6 \times 10^{-3} \mathrm{pb}\end{array}$ & $\begin{array}{l}\text { Fig. } 12 \text { of Ref. [22] } \\
5 \times 10^{-2} \mathrm{pb}\end{array}$ \\
\hline $\begin{array}{l}\tilde{\tau}^{ \pm} \tilde{\tau}^{\mp} \rightarrow 2\left(W^{ \pm} \rightarrow q q^{\prime}\right) \\
\quad+(\tilde{\nu} \rightarrow \ell \tau)+(\tilde{\nu} \rightarrow 2 \nu)\end{array}$ & $\ell+\tau+$ jets $+E_{\mathrm{T}}^{\mathrm{miss}}$ & $\begin{array}{l}(0.13 \mathrm{pb}) \times(0.6741)^{2} \times 2 \times \mathrm{BR}(\tilde{\nu} \rightarrow \ell \tau) \\
\quad \times\left(\frac{1}{3}\right) \times(0.1739) \times \mathrm{BR}(\tilde{\nu} \rightarrow 2 \nu) \\
\quad \leq 1.7 \times 10^{-3} \mathrm{pb}\end{array}$ & $\begin{array}{l}\text { Fig. } 12 \text { of Ref. [22] } \\
5 \times 10^{-2} \mathrm{pb}\end{array}$ \\
\hline $\begin{array}{l}\tilde{\tau}^{ \pm} \tilde{\tau}^{\mp} \rightarrow 2\left(W^{ \pm} \rightarrow q q^{\prime}\right) \\
\quad+2(\tilde{\nu} \rightarrow 2 \nu)\end{array}$ & jets $+E_{\mathrm{T}}^{\text {miss }}$ & $\begin{array}{l}(0.13 \mathrm{pb}) \times(0.6741)^{2} \times \mathrm{BR}(\tilde{\nu} \rightarrow 2 \nu) \\
\quad \leq 6 \times 10^{-2} \mathrm{pb}\end{array}$ & $\begin{array}{l}\text { Fig. } 20 \text { of Ref. [22] } \\
5 \times 10^{-1} \mathrm{pb}\end{array}$ \\
\hline
\end{tabular}

channel can be discussed in a similar way, and the BR to $e \mu$ fulfills $\operatorname{BR}\left(\tilde{\nu}_{\tau} \rightarrow e \mu\right) \approx \mathrm{BR}\left(\tilde{\nu}_{\tau} \rightarrow \mu \mu\right)+\operatorname{BR}\left(\tilde{\nu}_{\tau} \rightarrow e e\right)$ given that the BRs of the $\tau$ decays into electrons or muons (plus neutrinos) are similar $\approx 0.17$. To quantify roughly the value $\operatorname{BR}\left(\tilde{\nu}_{\tau} \rightarrow \mu \mu\right)$, we can use the following formula:

$$
\operatorname{BR}\left(\tilde{\nu}_{\tau} \rightarrow \mu \mu\right) \approx 0.068 \times\left(1+\frac{r}{3}\right)^{-1}
$$

with

$$
r \approx\left(\frac{\lambda}{Y_{\tau}}\right)^{2} \frac{2 m_{\nu}}{Y_{\nu}^{2} M}
$$

where we have neglected the first term in Eq. (2.1), which is a sensible approximation for small sneutrino masses around $50 \mathrm{GeV}$ provided that $\lambda$ is not large, and we have used the neutrino mass formula discussed below Eq. (1.4), $m_{\nu} \approx \sum_{i} v_{i}^{2} / 4 M$, implying that the decay width in Eq. (2.3) can be written as $\left(m_{\tilde{\nu}_{\tau}} / 16 \pi\right) 2 m_{\nu} / M$. In addition, we have set all neutrino Yukawas with a common value $Y_{\nu}$ in order to simplify the analysis. In what follows we will continue with this strategy, which does not essentially modify the results. Now, in the above equations and for typical values of the parameters such as e.g., $Y_{\nu}=5 \times 10^{-7}, m_{\nu}=$ $0.05 \mathrm{eV}$ and $M=1 \mathrm{TeV}$, one obtains $r=0.4$ and therefore a $\operatorname{BR}\left(\tilde{\nu}_{\tau} \rightarrow \mu \mu\right) \lesssim 0.06$ for $\lambda \gtrsim Y_{\tau}$.

In this approximation, we can also write the proper decay distance as

$$
\begin{aligned}
c \tau \approx & 0.22 \times\left(\frac{Y_{\nu}}{5 \times 10^{-7}}\right)^{-2}\left(\frac{\lambda}{Y_{\tau}}\right)^{2}\left(1+\frac{r}{3}\right)^{-1} \\
& \times\left(\frac{m_{\tilde{\nu}_{\tau}}}{60 \mathrm{GeV}}\right)^{-1} \mathrm{~mm}
\end{aligned}
$$

obtaining $c \tau \gtrsim 0.2 \mathrm{~mm}$ for $\lambda \gtrsim Y_{\tau}$. Thus the latter is a necessary condition on $\lambda$ in order to obtain suitable displaced vertices. In fact, as we will see in the next sections, we will need decay lengths larger than about a millimeter in order to be constrained by the current experimental results.

To compute numerically the mass spectrum and decay modes, we used a suitable modified version of the SARAH code [28] as well as the SPheno 3.3.6 code [29,30]. As an example, we show in Fig. 2(a) the BR for the decay of a scalar sneutrino $\tilde{\nu}_{\tau}^{\mathcal{R}}$ with a mass of $60 \mathrm{GeV}$ into $\mu \mu$, for 


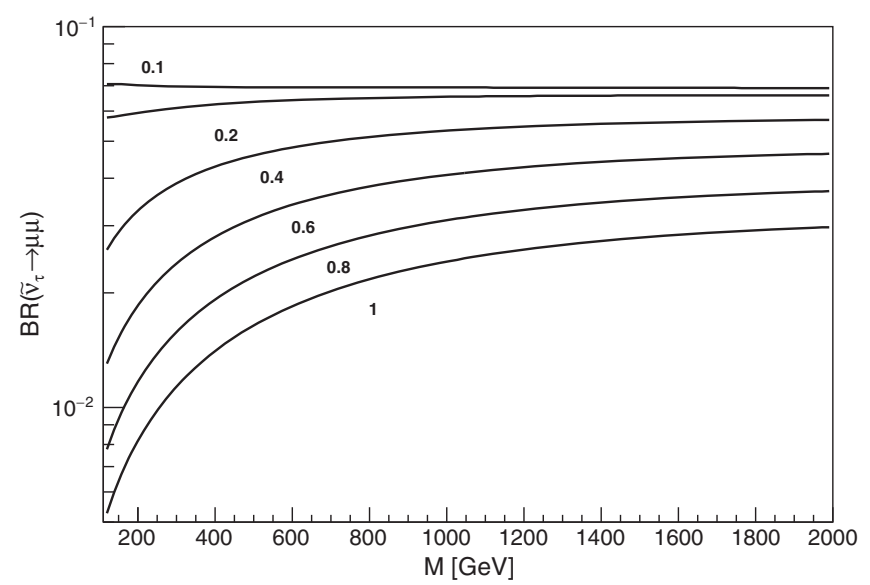

(a) Branching ratio

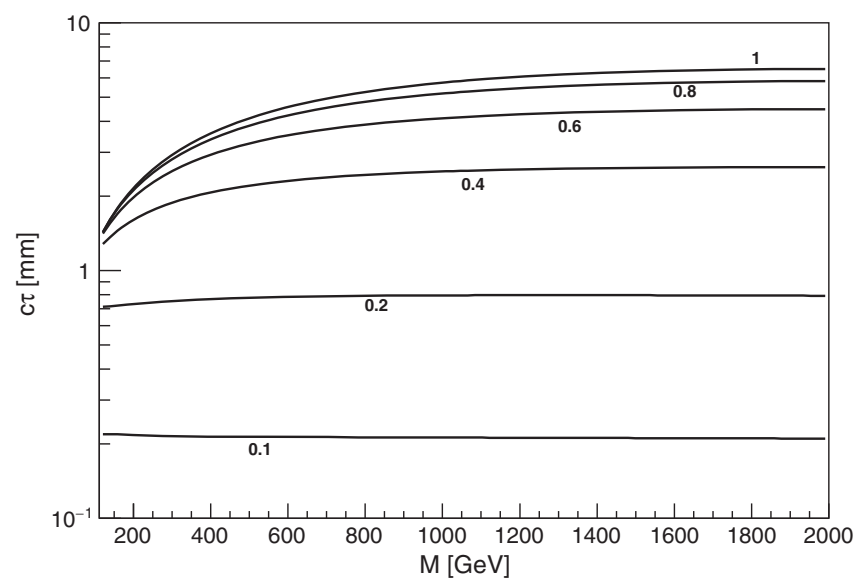

(b) Decay length

FIG. 2. (a) BR versus $M$ for the decay of a $\tilde{\nu}_{\tau}$ with $m_{\tilde{\nu}_{\tau}}=$ $60 \mathrm{GeV}$ into $\mu \mu$; (b) proper decay distance $c \tau$ of the $\tilde{\nu}_{\tau}$ versus $M$. In both plots (a) and (b), the neutrino Yukawas are set to $Y_{\nu}=5 \times 10^{-7}$, and several values of the coupling $\lambda$ are used such as $\lambda=0.1,0.2,0.4,0.6,0.8$, and 1 .

$Y_{\nu}=5 \times 10^{-7}$ (a similar figure is obtained in the case of the pseudoscalar $\tilde{\nu}_{\tau}^{\mathcal{I}}$ ). This is plotted as a function of $M$ for several values of the coupling $\lambda$. Values of $M$ smaller than $111.3 \mathrm{GeV}$ are not considered since the $\tilde{\nu}_{\tau}$ would no longer be the LSP in favor of the gauginos. Other parameters, whose effect on the sneutrino decay properties is less significant, as can be understood from previous formulas, are set to be $\kappa=0.3, \tan \beta=10$, and $\frac{v_{R}}{\sqrt{2}}=1350 \mathrm{GeV}$, throughout this work. Concerning the quantity $\sum_{i} v_{i}^{2}$ in Eq. (2.3), this is determined using Eq. (1.2) with the heavier neutrino mass fixed by the experimental constraints in the range $m_{\nu} \sim[0.05,0.23] \mathrm{eV}$, i.e., below the upper bound on the sum of neutrino masses $\sim 0.23 \mathrm{eV}$ [31], and above the square root of the mass-squared difference $\Delta m_{\mathrm{atm}}^{2} \sim 2.42 \times$ $10^{-3} \mathrm{eV}^{2}$ [32]. In Fig. 2(a) we chose as an example $m_{\nu}=0.05 \mathrm{eV}$. Finally, given the sneutrino mass formula in Eq. (1.5), there is enough freedom to tune $A_{\nu_{i}}$ in order to get the $\tilde{\nu}_{\tau}$ as the LSP with a mass of $60 \mathrm{GeV}$ as in the case of
Fig. 2(a). We can see in the figure that small values of $\lambda$ and large values of $M$ favor larger BRs to dileptons. These results can be easily deduced from Eqs. (2.4) and (2.5).

In Fig. 2(b), we show the proper decay distance $c \tau$ of the $\tilde{\nu}_{\tau}^{\mathcal{R}}$ for the same values of the parameters as in Fig. 2(a). Large values of $\lambda$ and $M$ favor larger decay lengths, as can be understood from Eqs. (2.6) and (2.5). As mentioned before, we need decay lengths larger than about a millimeter, and therefore for these values of the parameters the coupling is constrained to be $\lambda \gtrsim 0.2$. For example, for $\lambda=1$ we can see that the upper bound on the decay length is $c \tau=7 \mathrm{~mm}$. However, large values of $\lambda$ also favor smaller BRs into leptons, and therefore less stringent constraints on the parameter space. The interplay between these effects will be analyzed in the next sections. In addition, the values of $Y_{\nu}$ and $m_{\tilde{\nu}_{\tau}}$ also play an important role in the analysis. We can see in Eq. (2.6) that smaller values favor larger decay lengths. For example, for $m_{\tilde{\nu}_{\tau}}=60 \mathrm{GeV}$ as in Fig. 2 but $Y_{\nu}=10^{-7}$, we have checked that the upper bound on the decay length is $c \tau=20 \mathrm{~mm}$ for the case of $\lambda=1$. For $Y_{\nu}=$ $5 \times 10^{-7}$ as in Fig. 2 but $m_{\tilde{\nu}_{\tau}}=80 \mathrm{GeV}$, the upper bound for $\lambda=1$ turns out to be smaller as expected, $c \tau=4 \mathrm{~mm}$. Let us finally remark that $Y_{\nu}$ cannot be made arbitrarily small. Because of the relation $v_{i} \sim Y_{\nu} v_{u}$ discussed in the Introduction, this would imply that $v_{i}$ has to be also small coming into conflict with Eq. (1.2) and the constraint discussed above, $m_{\nu} \sim[0.05,0.23] \mathrm{eV}$. Thus a reasonable range for $Y_{\nu}$ is between $10^{-6}$ and $10^{-7}$.

\section{LONG-LIVED PARTICLE SEARCHES AT THE LHC}

As discussed in the previous section, a tau left sneutrino $\tilde{\nu}_{\tau}$ can decay into a pair of leptons with a proper lifetime of $\gtrsim 100 \mu \mathrm{m}$ [see Fig. 2(b)], long enough to have a visible separation from the production point. This signal can be compared with the searches for long-lived particles at the LHC.

There are various long-lived particle searches at the LHC, and each of them aims at a signature specific to a particular kind of particles. We thus first need to discuss which search strategy is most sensitive to $\tilde{\nu}_{\tau}$. Since they are electrically neutral, we are unable to use disappearing track searches or metastable charged particle searches to probe them. On the other hand, as we have seen above, the decay products of the sneutrinos include charged particles, and therefore we may detect the longevity of the sneutrinos by reconstructing their decay vertices, using the charged tracks associated with the daughter particles. This type of search strategies is dubbed as the displaced-vertex searches.

Both the ATLAS and CMS experiments have been searching for displaced vertices. The ATLAS $8-\mathrm{TeV}$ analysis [10] searches for events containing at least one long-lived particle decaying at a significant distance from the production point ( $\gtrsim 1 \mathrm{~mm}$ ), looking for decays into two 
leptons or into five or more charged particles. The latter search channel focuses on processes that produce a higher amount of charged particles and/or missing energy, compared with the decay of the $\tilde{\nu}_{\tau}$. We will however see below that the dilepton search channel in the ATLAS 8-TeV analysis is general enough as to be sensitive to the decay of the $\tilde{\nu}_{\tau}$. On the other hand, the current $13-\mathrm{TeV}$ displacedvertex search performed by the ATLAS collaboration [33] is optimized for long-lived gluinos, so it is not possible to use it for our purpose. As for the CMS, the $8-\mathrm{TeV}$ analysis presented in Ref. [11] gives a limit comparable to the ATLAS 8-TeV bound if the decay distance of sneutrinos is $\gtrsim 3 \mathrm{~cm}$-although this CMS search is in principle sensitive to shorter decay distances as the selection cut requires $\left|d_{0}\right|<12 \sigma_{d} \sim 180 \mu \mathrm{m}\left(d_{0}\right.$ is the transverse impact parameter of tracks and $\sigma_{d}$ is its uncertainty), their limits are terminated at a much larger value of $c \tau$ especially for soft displaced vertices (see, e.g., Fig. 6 in Ref. [11]). Given that the ATLAS 8-TeV analysis provides bounds for smaller $c \tau$ compared with those from the CMS study, to make the discussions concrete, we focus on displaced-vertex searches with the ATLAS detector in what follows.

The ATLAS displaced-vertex search in Ref. [10] is based on the 8-TeV data with an integrated luminosity of $20.3 \mathrm{fb}^{-1}$. Among the various search channels studied in the analysis, the dilepton displaced-vertex selection channel, where each displaced vertex is formed from at least two oppositely charged leptons, may be used for the long-lived $\tilde{\nu}_{\tau}$ search. As we mentioned above, we focus on the decay processes of $\tilde{\nu}_{\tau}$ in which $\tau$ leptons in the final state decay into leptons, in order to utilize this selection channel.

In the dilepton displaced-vertex search, each event must satisfy the muon or electron trigger requirement. ${ }^{2}$ For the muon trigger, a muon candidate is identified only in the muon spectrometer, without utilizing the tracking information, and required to have a transverse momentum of $p_{\mathrm{T}}>$ $50 \mathrm{GeV}$ and the pseudorapidity of $|\eta|<1.07$. For the electron trigger, only a high-energy deposit in the electromagnetic calorimeter is required, again without tracker requirements. This has a less effective background rejection performance compared with the muon trigger, and thus a relatively strong criterion is imposed on the transverse momentum of electrons: either a single electron with $p_{\mathrm{T}}>$ $120 \mathrm{GeV}$ or two electrons with $p_{\mathrm{T}}>40 \mathrm{GeV}$. The events which have passed these triggers are then required to be subject to the object reconstruction and filtering criteria. Finally, with the help of the retracking procedure, a dilepton displaced vertex is reconstructed from two oppositely charged lepton tracks: $\mu^{+} \mu^{-}, e^{+} e^{-}$, or $e^{ \pm} \mu^{\mp}$. Here, the lepton tracks are required to satisfy $p_{\mathrm{T}}>10 \mathrm{GeV}, 0.02 \leq$ $|\eta|<2.5$, and $d_{0}>2 \mathrm{~mm}(2.5 \mathrm{~mm})$ for muons (electrons). In addition, the invariant mass of the tracks, $m_{\mathrm{DV}}$, should be

\footnotetext{
${ }^{2}$ In the ATLAS search, the missing-energy and jets triggers are also used. These triggers are ineffective in our setup.
}

larger than $10 \mathrm{GeV}$. The position of the reconstructed displaced vertices must satisfy $r_{\mathrm{DV}}<300 \mathrm{~mm},\left|z_{\mathrm{DV}}\right|<$ $300 \mathrm{~mm}$, and $\sqrt{\left(x_{\mathrm{DV}}-x_{\mathrm{PV}}\right)^{2}+\left(y_{\mathrm{DV}}-y_{\mathrm{PV}}\right)^{2}} \geq 4 \mathrm{~mm}$, where the subscripts DV and PV indicate that the corresponding coordinates are those of the displaced vertex and the primary vertex, respectively. The effect of the first two conditions is almost negligible in our analysis since the decay distance of sneutrinos is $\lesssim 10 \mathrm{~mm}$ as shown in Fig. 2(b), while the third condition does affect our analysis, as we shall see below.

With these requirements, the ATLAS collaboration searched for dilepton displaced vertices and found no event, while the numbers of background events are expected to be $1.0 \pm 0.2_{-0.6}^{+0.3} \times 10^{-3}, 2.4 \pm 0.9_{-1.5}^{+0.8} \times 10^{-3}$, and $2.0 \pm 0.5_{-1.4}^{+0.3} \times 10^{-3}$ for the $e^{+} e^{-}, e^{ \pm} \mu^{\mp}$, and $\mu^{+} \mu^{-}$ channels, respectively. The dominant source of the background is accidental crossings of independent lepton tracks. As we see, this search is basically background free. With this result, strong limits were imposed on long-lived particles which decay into leptons.

We however cannot directly apply the limits provided by the ATLAS collaboration (Fig. 13 of Ref. [10]) to the left sneutrino case, since the ATLAS analysis simulates the decay of a heavy gluino into a light and a heavy neutralino. The former case represents a highly boosted light particle decaying into a pair of muons, while the latter represents a heavy nonboosted particle decaying in the same way. Yet, the sneutrino features a light nonboosted particle. This analysis can be extended, nevertheless, combining information from both situations by considering the fact that the difference in the strength of the upper limits basically comes from the efficiency in passing the event selection requirements (the decay products from a sufficiently heavy neutralino are so energetic that almost all the events pass the selection criteria), ${ }^{3}$ while the position of the minimum of the limits is determined by the boost factors of the neutralinos. Thus, what we can do is shifting upwards the limit corresponding to the nonboosted neutralino to make its minimum to coincide with the one of the line corresponding to the light boosted neutralino. ${ }^{4}$ The resultant limit for the dimuon channel is displayed as a function of the decay distance $c \tau$ in the purple-shaded solid line in

\footnotetext{
${ }^{3}$ In the ATLAS search [10], the light neutralino events can pass the trigger requirement since the missing-energy and jets triggers are also used, but the vertex-level efficiency deteriorates for such events. With keeping this in mind, we here assume that all of the neutralino events satisfy the trigger requirements-the dilepton trigger for the heavy case and the missing-energy/jets trigger for the light case - and the difference in the sensitivities originates from that in the vertex-level efficiencies. This assumption is fairly reasonable since the leptons (jets) in the final state are very active in the heavy (light) neutralino case.

${ }^{4}$ We however note that there is also an efficiency loss for a boosted system as the final state muons tend to be collinear with each other, whose effect is not taken into account in this prescription. The neglect of this effect thus results in a rather conservative limit.
} 


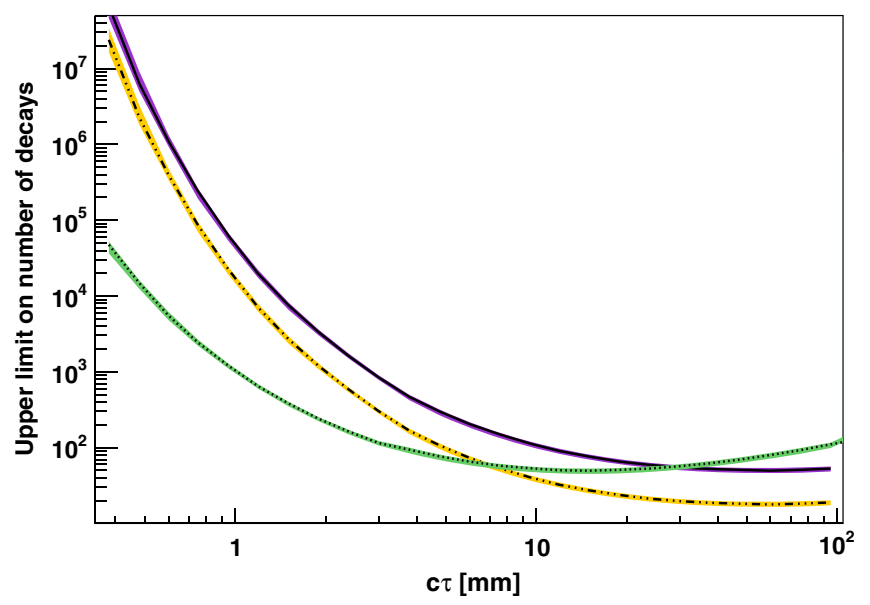

FIG. 3. Upper limit at $95 \%$ confident level on the number of tau left sneutrinos decaying into dimuons at $\sqrt{s}=8 \mathrm{TeV}$ for an integrated luminosity of $20.3 \mathrm{fb}^{-1}$, shown in the purple-shaded solid line. We also show the limits given in Fig. 13(c) of Ref. [10], which we use to obtain our limit: green-hatched dotted (yellowhatched dash-dotted) line corresponding to a light boosted neutralino of $50 \mathrm{GeV}$ (heavy nonboosted neutralino of $1000 \mathrm{GeV}$ ) from the decay of a heavy gluino of $1300 \mathrm{GeV}$.

Fig. 3. We also show the limits corresponding to the light boosted and heavy nonboosted cases in the green-hatched dotted and yellow-hatched dash-dotted lines, respectively, which are taken from Fig. 13(c) of Ref. [10]. We analyzed the limits for the $e e$ and $e \mu$ channels in a similar manner using Figs. 13(a) and 13(b) in Ref. [10], respectively, obtaining very similar plots. As seen from Fig. 3, the ATLAS displaced-vertex search is sensitive to a decay distance larger than about a few $\mathrm{mm}$. This stems from the requirements that the impact parameter $d_{0}$ of the muon tracks be larger than $2 \mathrm{~mm}$ and the transverse distance between the displaced vertices and the primary vertices be larger than $4 \mathrm{~mm}$, as we mentioned above. We will discuss a possibility of relaxing these requirements later.

Another obstacle for the left sneutrino case is the trigger requirement. Since left sneutrinos we consider in this paper have a mass of $\lesssim 100 \mathrm{GeV}$ and are less boosted, their decay products have relatively small momenta. On the other hand, the ATLAS 8-TeV analysis requires rather high thresholds for lepton momenta, especially for electrons, since it aims at generic long-lived particles such as metastable neutrinos produced by the decays of colored particles. In particular, the events must satisfy the following requirements [10]:

(i) One muon with $p_{\mathrm{T}}>50 \mathrm{GeV}$ and $|\eta|<1.07$, one electron with $p_{\mathrm{T}}>120 \mathrm{GeV}$ or two electrons with $p_{\mathrm{T}}>40 \mathrm{GeV}$.

(ii) One pair $e^{+} e^{-}, \mu^{+} \mu^{-}$or $e^{ \pm} \mu^{\mp}$ with $p_{\mathrm{T}}>10 \mathrm{GeV}$ and $0.02<|\eta|<2.5$ for each one.

To estimate the sensitivity of this search strategy, samples of simulated Monte Carlo (MC) events are used to study the efficiency of the triggering and off-line selection processes for signal events. In each event two sneutrinos are created in the $p p$ collision as described in Sec. II. All simulated samples are generated using MadGraph5_aMC@NLO 2.6.0 [34] and PYTHIA 8.230 [35]. In particular, 10,000 events are generated for each case with MadGraph5_aMC@NLO 2.6.0 at leading order (LO) of perturbative QCD simulating the production of the described process. We include the nextto-leading order (NLO) [16] and next-to-leading logarithmic accuracy [36] results using a $K$-factor of about 1.2. We also use DELPHES v3 [37] for the detector simulation. The effect of these selections for different masses and for the three production processes at $\sqrt{s}=8 \mathrm{TeV}$ is shown in Tables III and IV for the $\mu \mu$ and $\mu e$ channels, respectively. The trigger requirement for electrons is too restrictive and makes the selection efficiency for the dielectron channel to be a few percent level, and thus ineffective for light sneutrino searches. In these tables, $\epsilon_{\text {sel }}$ is the selection efficiency for each case. Using these results and the BRs discussed in Sec. II, we can compute the prediction for the number of decays $\tilde{\nu}_{\tau} \rightarrow \mu \mu / \mu e$ passing the trigger and event selection requirements. Notice however that $\epsilon_{\text {sel }}$ is not large.

The ATLAS 8-TeV search strategy discussed above is, of course, not optimized for the present setup. Nevertheless, in principle it is possible to optimize the trigger requirements for left sneutrino searches by relaxing the thresholds, as there are a variety of different lepton triggers with lower momentum thresholds used in the ATLAS experiment. For instance, for the muon trigger, the ATLAS 8-TeV analysis uses only the muon spectrometer and requires $p_{\mathrm{T}}>50 \mathrm{GeV}$, as discussed above. On the other hand, the MU24I trigger [38], which is an isolated single muon trigger at the event filter, also uses the information from the inner detector and requires the transverse momentum threshold of $p_{\mathrm{T}}>24 \mathrm{GeV}$. With the help of the inner detector information, this MU24I trigger has a good performance in a wider range of the pseudorapidity of tracks, and thus we can also relax the requirement on $\eta$; from $|\eta|<1.07$ to $|\eta|<2.5$ [38]. To exploit this trigger instead of that used in Ref. [10] can significantly enhance the sensitivity to light sneutrinos, since the typical momentum of muons from the sneutrino decays is a few tens of GeV. A side effect of the reduction of the momentum threshold is, of course, an increase of the number of background events. According to Ref. [38], the enhancement in the number of events due to the relaxation of the trigger requirement is expected to be

\footnotetext{
${ }^{5}$ This trigger should also satisfy a loose isolation selection, the sum of the $p_{\mathrm{T}}$ of tracks in a cone of $\Delta R<0.2$ centered around the muon candidate after eliminating the muon transverse momentum $\left(p_{\mathrm{T}}\right)_{\mu}$ should be smaller than $0.12 \times\left(p_{\mathrm{T}}\right)_{\mu}$; this requirement is so loose that almost all isolated muons from the $Z$-boson decays pass the criterion. Since the muons coming from the sneutrino decays are also expected to be isolated, we can expect that this requirement scarcely affects the sneutrino event selection. For this reason, we do not take account this effect in the following analysis.
} 
TABLE III. Cutflow of the selection requirements for $\tilde{\nu}_{\tau} \rightarrow \mu \mu$ for the 8-TeV analysis, where $\epsilon_{\text {sel }}$ is the selection efficiency. For each case, 10,000 MC events are generated.

\begin{tabular}{lrrrrrrrrrrrr}
\hline \hline & \multicolumn{3}{c}{ Through $Z$ boson } & \multicolumn{4}{c}{ Through $W$ boson } & \multicolumn{3}{c}{ Through $\gamma, Z$ bosons } \\
\hline$m_{\tilde{\nu}_{\tau}}(\mathrm{GeV})$ & 50 & 60 & 80 & 100 & 50 & 60 & 80 & 100 & 50 & 60 & 80 & 100 \\
$p_{T_{\text {triger }}}$ & 2,280 & 4,241 & 6,828 & 8,063 & 2,335 & 3,703 & 3,840 & 7,515 & 1,616 & 2,233 & 3,396 & 4,374 \\
Dilepton selection & 1,069 & 2,096 & 3,517 & 4,333 & 1,135 & 1,881 & 3,740 & 3,968 & 1,550 & 2,150 & 3,273 & 4,208 \\
$\epsilon_{\text {sel }}$ & 0.1069 & 0.2096 & 0.3517 & 0.4333 & 0.1135 & 0.1881 & 0.374 & 0.3968 & 0.1550 & 0.2150 & 0.3273 & 0.4208 \\
\hline \hline
\end{tabular}

TABLE IV. The same as in Table III but for $\tilde{\nu}_{\tau} \rightarrow \mu e$.

\begin{tabular}{|c|c|c|c|c|c|c|c|c|c|c|c|c|}
\hline \multirow[b]{2}{*}{$m_{\tilde{\nu}_{\tau}}(\mathrm{GeV})$} & \multicolumn{4}{|c|}{ Through $Z$ boson } & \multicolumn{4}{|c|}{ Through $W$ boson } & \multicolumn{4}{|c|}{ Through $\gamma, Z$ bosons } \\
\hline & 50 & 60 & 80 & 100 & 50 & 60 & 80 & 100 & 50 & 60 & 80 & 100 \\
\hline$p_{\text {Ttrigger }}$ & 659 & 1,347 & 2,526 & 3,612 & 678 & 1,138 & 2,134 & 3,067 & 878 & 1,249 & 2,329 & 3,287 \\
\hline Dilepton selection & 618 & 1,253 & 2,315 & 3,278 & 628 & 1,061 & 1,972 & 2,803 & 827 & 1,160 & 2,109 & 2,978 \\
\hline$\epsilon_{\mathrm{sel}}$ & 0.0618 & 0.1253 & 0.2315 & 0.3278 & 0.0628 & 0.1061 & 0.1972 & 0.2803 & 0.0827 & 0.116 & 0.2109 & 0.2978 \\
\hline
\end{tabular}

10. Since the main background in the displaced-vertex search is accidental crossings of uncorrelated lepton tracks [10], we can estimate the increase in the number of background events by scaling this according to the number of events passing the trigger. Given that the number of background muon vertices in the ATLAS $8-\mathrm{TeV}$ search is as low as $\sim 2 \times 10^{-3}$ [10], we can safely conclude that the number of background events can still be regarded as zero even if we relax the trigger requirement for muons. Another restriction we need to take into account is the requirement on the impact parameter $d_{0}$ of muon tracks adopted by MU24I; $\left|d_{0}\right|<1 \mathrm{~cm}$ is required $[39,40]$ for the MU24I trigger, which indicates that the efficiency should be reduced for sneutrinos with $c \tau \gtrsim 1 \mathrm{~cm}$. Nevertheless, this again causes a negligible effect on left sneutrino searches in the present setup since the sneutrinos have a proper decay distance smaller than $1 \mathrm{~cm},{ }^{6}$ as shown in Fig. 2(b). We therefore conclude that the use of the MU24I trigger instead of the present one in Ref. [10] is very powerful and promising for the left sneutrino searches.

We may also use a lower $p_{\mathrm{T}}$ threshold for the electron trigger. However, we are unable to estimate the increase in the number of background events in this case from, say, Ref. [41], since the plot does not show the corresponding trigger rate for $p_{\mathrm{T}}>120 \mathrm{GeV}$. Considering this, in the following $8-\mathrm{TeV}$ analysis, we only use the muon trigger with $p_{\mathrm{T}}>24 \mathrm{GeV}$ and consider the $\mu^{+} \mu^{-}$and $\mu^{ \pm} e^{\mp}$ channels to be conservative. We however note that we can certainly optimize the electron trigger as well, which indeed improves the sensitivity considerably and thus is worth a further dedicated study.

After all, we use the following criteria for the optimized 8-TeV analysis:

\footnotetext{
${ }^{6}$ This is the reason why we show only a small $c \tau$ region in Fig. 3.
}

(i) At least one muon with $p_{\mathrm{T}}>24 \mathrm{GeV}$.

(ii) One pair $\mu^{+} \mu^{-}$or $e^{ \pm} \mu^{\mp}$ with $p_{\mathrm{T}}>10 \mathrm{GeV}$ and $0.02<|\eta|<2.5$ for each one.

The effect of these selections for different masses and for the three production processes, is shown in Tables V and VI for the $\mu \mu$ and $\mu e$ channels, respectively. We see that a sizable number of signal events is expected to pass the selection criteria. We can compare these results with those of Table III. For instance, there $\epsilon_{\text {sel }} \simeq 0.11$ is obtained for a $50-\mathrm{GeV}$ sneutrino produced via a $Z$-boson, whereas $\epsilon_{\mathrm{sel}} \simeq$ 0.66 is obtained in Table $\mathrm{V}$, with a significant improvement in the event selection.

We also study the prospects for the 13-TeV LHC run. Since we do not have any dedicated searches for dilepton displaced vertices with the $13-\mathrm{TeV}$ data so far, we just assume background-free in our estimation. Again, we consider an optimization of the trigger requirements in the $13-\mathrm{TeV}$ analysis using the existing result for the performance of the ATLAS trigger system [42], taking account of the trigger rate for the $8-\mathrm{TeV}$ analysis. Since the trigger rate for MU24I is $\lesssim 100 \mathrm{~Hz}$ [38], we expect a sufficiently low background as long as the trigger rate in the $13-\mathrm{TeV}$ searches does not exceed about $100 \mathrm{~Hz}$. According to Ref. [42], a $p_{\mathrm{T}}$ threshold of $26 \mathrm{GeV}[43,44]$ for both muon and electron ensures the trigger rate to be $\lesssim 100 \mathrm{~Hz}^{7}$ These triggers again rely on the use of the inner tracker, ${ }^{8}$ and thus are effective in the region of $|\eta|<2.5$. With this observation, we use the following criteria for the $13-\mathrm{TeV}$ analysis:

(i) At least one electron or muon with $p_{\mathrm{T}}>26 \mathrm{GeV}$.

(ii) One pair $\mu^{+} \mu^{-}, e^{+} e^{-}$, or $e^{ \pm} \mu^{\mp}$ with $p_{\mathrm{T}}>10 \mathrm{GeV}$ and $0.02<|\eta|<2.5$ for each one.

\footnotetext{
${ }^{7}$ Given a higher instantaneous luminosity $\left(\sim 2 \times 10^{34} \mathrm{~cm}^{-2} \mathrm{~s}^{-1}\right)$ compared with those considered in Ref. [42], the momentum threshold is raised from the ones in Ref. [42] so that the trigger rates are kept at a similar level.

${ }^{8}$ For the tracking performance in the $13 \mathrm{TeV}$ run, see Ref. [45].
} 
TABLE V. Cutflow of the selection requirements for $\tilde{\nu}_{\tau} \rightarrow \mu \mu$ for the optimized 8-TeV analysis, where $\epsilon_{\text {sel }}$ is the selection efficiency. For each case, 10,000 MC events are generated.

\begin{tabular}{lcccccccccccc}
\hline \hline & \multicolumn{3}{c}{ Through $Z$ boson } & \multicolumn{3}{c}{ Through $W$ boson } & \multicolumn{4}{c}{ Through $\gamma, Z$ bosons } \\
\hline$m_{\tilde{\nu}_{\tau}}(\mathrm{GeV})$ & 50 & 60 & 80 & 100 & 50 & 60 & 80 & 100 & 50 & 60 & 80 & 100 \\
$p_{\text {Ttrigger }}$ & 6,687 & 7,775 & 8,682 & 9,144 & 6,520 & 7,343 & 7,359 & 8,846 & 6,961 & 7,471 & 8,387 & 8,916 \\
Dilepton selection & 6,637 & 7,707 & 8,589 & 9,027 & 6,409 & 7,198 & 7,229 & 8,594 & 6,701 & 7,288 & 8,151 & 8,647 \\
$\epsilon_{\text {sel }}$ & 0.6637 & 0.7707 & 0.8589 & 0.9027 & 0.6409 & 0.7198 & 0.7229 & 0.8594 & 0.6701 & 0.7288 & 0.8151 & 0.8647 \\
\hline \hline
\end{tabular}

TABLE VI. The same as in Table $\mathrm{V}$ but for $\tilde{\nu}_{\tau} \rightarrow \mu e$.

\begin{tabular}{lccccccccccccc}
\hline \hline & \multicolumn{3}{c}{ Through $Z$ boson } & \multicolumn{3}{c}{ Through $W$ boson } & \multicolumn{4}{c}{ Through $\gamma, Z$ bosons } \\
\hline$m_{\tilde{\nu}_{\tau}}(\mathrm{GeV})$ & 50 & 60 & 80 & 100 & 50 & 60 & 80 & 100 & 50 & 60 & 80 & 100 \\
$p_{\text {Ttrigger }}$ & 4,014 & 5,255 & 6,508 & 7,420 & 3,853 & 4,754 & 6,054 & 6,974 & 6,118 & 6,748 & 7,116 & 8,293 \\
Dilepton selection & 3,943 & 5,094 & 6,267 & 7,067 & 3,763 & 4,624 & 5,830 & 6,637 & 4,917 & 5,515 & 6,360 & 6,961 \\
$\epsilon_{\text {sel }}$ & 0.3943 & 0.5094 & 0.6267 & 0.7067 & 0.3763 & 0.4624 & 0.5830 & 0.6637 & 0.4917 & 0.5515 & 0.6360 & 0.6916 \\
\hline \hline
\end{tabular}

TABLE VII. Cutflow of the selection requirements for $\tilde{\nu}_{\tau} \rightarrow \mu \mu$, for the optimized 13-TeV analysis, where $\epsilon_{\text {sel }}$ is the selection efficiency. For each case, 10,000 MC events are generated.

\begin{tabular}{lrrrrrrrrrrrr}
\hline & \multicolumn{3}{c}{ Through $Z$ boson } & \multicolumn{3}{c}{ Through $W$ boson } & \multicolumn{3}{c}{ Through $\gamma, Z$ bosons } \\
\hline$m_{\tilde{\nu}_{\tau}}(\mathrm{GeV})$ & 50 & 60 & 80 & 100 & 50 & 60 & 80 & 100 & 50 & 60 & 80 & 100 \\
$p_{\text {Ttrigger }}$ & 5,797 & 7,011 & 8,077 & 8,581 & 5,643 & 6,539 & 7,587 & 8,281 & 5,885 & 6,634 & 7,716 & 8,316 \\
Dilepton selection & 5,739 & 6,941 & 7,995 & 8,469 & 5,587 & 6,411 & 7,384 & 8,020 & 5,705 & 6,459 & 7,485 & 8,000 \\
$\epsilon_{\text {sel }}$ & 0.5739 & 0.6941 & 0.7995 & 0.8469 & 0.5587 & 0.6411 & 0.7384 & 0.8020 & 0.5705 & 0.6459 & 0.7485 & 0.8 \\
\hline \hline
\end{tabular}

TABLE VIII. The same as in Table VII but for $\tilde{\nu}_{\tau} \rightarrow \mu e$.

\begin{tabular}{lrrrrlrrrrrrr}
\hline \hline & \multicolumn{3}{c}{ Through $Z$ boson } & \multicolumn{3}{c}{ Through $W$ boson } & \multicolumn{4}{c}{ Through $\gamma, Z$ bosons } \\
\hline$m_{\tilde{\nu}_{\tau}}(\mathrm{GeV})$ & 50 & 60 & 80 & 100 & 50 & 60 & 80 & 100 & 50 & 60 & 80 & 100 \\
$p_{\text {Ttrigger }}$ & 5,344 & 6,386 & 7,458 & 8,149 & 4,929 & 5,751 & 6,961 & 7,698 & 6,836 & 5,971 & 6,912 & 7,698 \\
Dilepton selection & 4,312 & 5,203 & 6,138 & 6,718 & 3,901 & 4,652 & 5,647 & 6,226 & 3,089 & 4,883 & 5,629 & 6,257 \\
$\epsilon_{\text {sel }}$ & 0.4312 & 0.5203 & 0.6138 & 0.6718 & 0.3901 & 0.4652 & 0.5647 & 0.6226 & 0.3089 & 0.4883 & 0.5629 & 0.6257 \\
\hline \hline
\end{tabular}

TABLE IX. The same as in Table VII but for $\tilde{\nu}_{\tau} \rightarrow e e$.

\begin{tabular}{lrrrrrrrrrrrr}
\hline \hline & \multicolumn{3}{c}{ Through $Z$ boson } & \multicolumn{3}{c}{ Through $W$ boson } & \multicolumn{3}{c}{ Through $\gamma, Z$ bosons } \\
\hline$m_{\tilde{\nu}_{\tau}}(\mathrm{GeV})$ & 50 & 60 & 80 & 100 & 50 & 60 & 80 & 100 & 50 & 60 & 80 & 100 \\
$p_{\text {Ttrigger }}$ & 4,724 & 5,766 & 6,892 & 7,633 & 4,177 & 4,943 & 6,046 & 6,750 & 4,414 & 5,132 & 6,078 & 6,836 \\
Dilepton selection & 1,886 & 2,413 & 3,011 & 3,428 & 1,627 & 1,996 & 2,476 & 2,797 & 1,868 & 2,200 & 2,739 & 3,089 \\
$\epsilon_{\text {sel }}$ & 0.1886 & 0.2413 & 0.3011 & 0.3428 & 0.1627 & 0.1996 & 0.2476 & 0.2797 & 0.1868 & 0.2200 & 0.2739 & 0.3089 \\
\hline \hline
\end{tabular}

The effect of these selections for different masses and for the three production processes at $\sqrt{s}=13 \mathrm{TeV}$ is shown in Tables VII-IX for the $\mu \mu, \mu e$, and $e e$ channels, respectively. ${ }^{9}$ We however note that a more elaborate optimization may be considered; e.g., we may also use the dilepton triggers,

\footnotetext{
${ }^{9}$ The efficiency of the $e e$ channel is worse than the other channels due to the isolation requirement for electrons implemented in the detector simulation with DELPHES v3 [37].
}

which may be more effective since we can lower the momentum threshold for these triggers [42]. In any case, to use a high level trigger with inner-detector information is technically quite feasible and expected to result in a considerable improvement in displaced-vertex searches. We also note in passing that this possible improvement is not only for the ATLAS analysis but also for the CMS one [11], where again tracker information is not used in the trigger requirement. 
Now we discuss how to obtain the limits for light sneutrinos. The limits from the ATLAS search [10] can be translated into a vertex-level efficiency, taking into account the lack of observation of events for any value of the decay length. Therefore, $\epsilon_{\text {vert }}(c \tau)$ can be obtained as the ratio of the number of signal events compatible with zero observed events (which in this case is 3) and that corresponding to the upper limits given in Ref. [10] (with an appropriate modification described above); e.g., we can use the purple-shaded solid line of Fig. 3 to obtain the vertex-level efficiency $\epsilon_{\text {vert }}^{\mu \mu}(c \tau)$ for the dimuon channel. By multiplying the number of the events passing the trigger and event selection criteria, which is computed above, with this vertex-level efficiency, we can estimate the total number of signal events; for the $8-\mathrm{TeV}$ case, this is given for the $\mu \mu$ channel by

$$
\begin{aligned}
\text { \#Dimuons }= & {\left[\sigma\left(p p \rightarrow Z \rightarrow \tilde{\nu}_{\tau} \tilde{\nu}_{\tau}\right) \epsilon_{\mathrm{sel}}^{Z}\right.} \\
& +\sigma\left(p p \rightarrow W \rightarrow \tilde{\nu}_{\tau} \tilde{\tau}\right) \epsilon_{\mathrm{sel}}^{W} \\
& \left.+\sigma(p p \rightarrow \gamma, Z \rightarrow \tilde{\tau} \tilde{\tau}) \epsilon_{\mathrm{sel}}^{\gamma, Z}\right] \\
& \times \mathcal{L} \times\left[\operatorname{BR}\left(\tilde{\nu}_{\tau}^{\mathcal{R}} \rightarrow \mu \mu\right) \epsilon_{\mathrm{vert}}^{\mu \mu}\left(c \tau^{\mathcal{R}}\right)\right. \\
& \left.+\operatorname{BR}\left(\tilde{\nu}_{\tau}^{\mathcal{I}} \rightarrow \mu \mu\right) \epsilon_{\mathrm{vert}}^{\mu \mu}\left(c \tau^{\mathcal{I}}\right)\right],
\end{aligned}
$$

where the selection efficiencies $\epsilon_{\mathrm{sel}}^{Z}, \epsilon_{\mathrm{sel}}^{W}$ and $\epsilon_{\mathrm{sel}}^{\gamma, Z}$ are given in Tables III and V. The same formula can be applied for the $e \mu$ channel shown in Tables IV and VI, using the corresponding BRs, selection efficiencies, and vertex-level efficiencies (which turn out to be similar). For the $13-\mathrm{TeV}$ prospects the selection efficiencies for the three channels can be found in Tables VII-IX, and we use the same vertex-level efficiency as in the 8-TeV case and assume zero background. ${ }^{10}$ As a result, if this predicted number of signal events is above 3 the corresponding parameter point of the model is excluded so that this is compatible with zero number of events.

We note in passing that the optimization strategy we have discussed in this section is generically useful for the searches of other metastable particles which have a relatively short lifetime and a small mass and whose decay products contain soft leptons. We thus hope this kind of search strategies is considered seriously in the LHC experiments.

Another possibility to improve the sensitivity is to search for shorter displaced vertices. As can be seen from Fig. 2(b), in some regions of parameter space, the decay distance of a sneutrino is predicted to be $\lesssim 1 \mathrm{~mm}$, to which the current ATLAS search is less sensitive. However, we may even probe such submillimeter region by relaxing the impact-

\footnotetext{
${ }^{10}$ Notice that in the $13-\mathrm{TeV}$ long-lived gluino search [33] the estimated number of background events is still much smaller than one, $\sim 10^{-2}$, which is similar in size to that in the $8-\mathrm{TeV}$ search [10]. We therefore expect that the background in the $13-\mathrm{TeV}$ dilepton displaced-vertex search is also as low as the 8-TeV one.
}

parameter requirements for lepton tracks as well as the condition on the reconstructed position of displaced vertices, given the extremely low background in this search [10]. In fact, it is shown in Refs. [46,47] for metastable gluinos that submillimeter displaced vertices can be probed using the existing vertex-reconstruction technique, though this is not directly applicable to the present case. Moreover, there are several existing searches which are sensitive to the submillimeter region $[11,48-50]$. In any case, to assess the possibility of searching for shorter dilepton displaced vertices, a dedicated study with a full consideration of the detector performance is required; we thus do not discuss this possibility in this paper and hand this over to experimentalists.

\section{RESULTS}

By using the method described in the previous section, we now evaluate the current and potential limits on the parameter space of our scenario from the displaced-vertex searches with the 8-TeV ATLAS result [10], and discuss the prospects for the $13-\mathrm{TeV}$ searches.

The 8-TeV current limits are given in Figs. 4 and 5, with the neutrino mass scale fixed to be 0.05 and $0.23 \mathrm{eV}$, respectively, and all of the neutrino Yukawa couplings set to be a common value $Y_{\nu}: 10^{-7}, 5 \times 10^{-7}$, and $10^{-6} \mathrm{GeV}$ in the top, middle, and bottom panels, respectively. The yellow, red, blue, and green lines correspond to the sneutrino mass of $50,60,80$, and $100 \mathrm{GeV}$, respectively, with the bands representing the uncertainties that come from those in Ref. [10], i.e., the $\pm 1 \sigma$ variations in the expected limit in Fig. 13(c) of the aforementioned reference. Notice that for these sneutrino masses, values of $M$ smaller than 92.7, 111.3, 148.4, and $185.5 \mathrm{GeV}$, respectively, are not interesting for our analysis since the tau left sneutrino would no longer be the LSP in favor of the gauginos. To obtain the reaches, we have combined the results from the $\mu \mu$ and $e \mu$ channels. The region of the parameter space to the right and above each line is excluded from the displaced-vertex searches.

We can see in the top panel of Fig. 4, where $Y_{\nu}=10^{-7}$, that for $m_{\tilde{\tau}_{\tau}}=50 \mathrm{GeV}$ the upper bound on the average gaugino mass $M$ [see Eq. (1.4)] is of about 500 (900) GeV for $\lambda=0.1$ (1). As discussed in Sec. II, small values of $\lambda$ favor larger BRs, and as a consequence the gaugino mass is more constrained. If $\lambda$ is too small, however, the limit on $M$ disappears since the lifetime of the left sneutrino goes into the submillimeter regime, as can be seen from Eq. (2.6). On the other hand, small sneutrino masses produce larger decay lengths, and gaugino masses turn out to be also more constrained. For example, for $m_{\tilde{\nu}_{\tau}}=50$ and $60 \mathrm{GeV}$ the upper bound on $M$ for $\lambda=1$ is of about 700 and $1100 \mathrm{GeV}$, respectively. In the middle panel, the larger value of the neutrino Yukawa $Y_{\nu}=5 \times 10^{-7}$ gives rise to smaller decay lengths, and therefore the figures are shifted to the right 

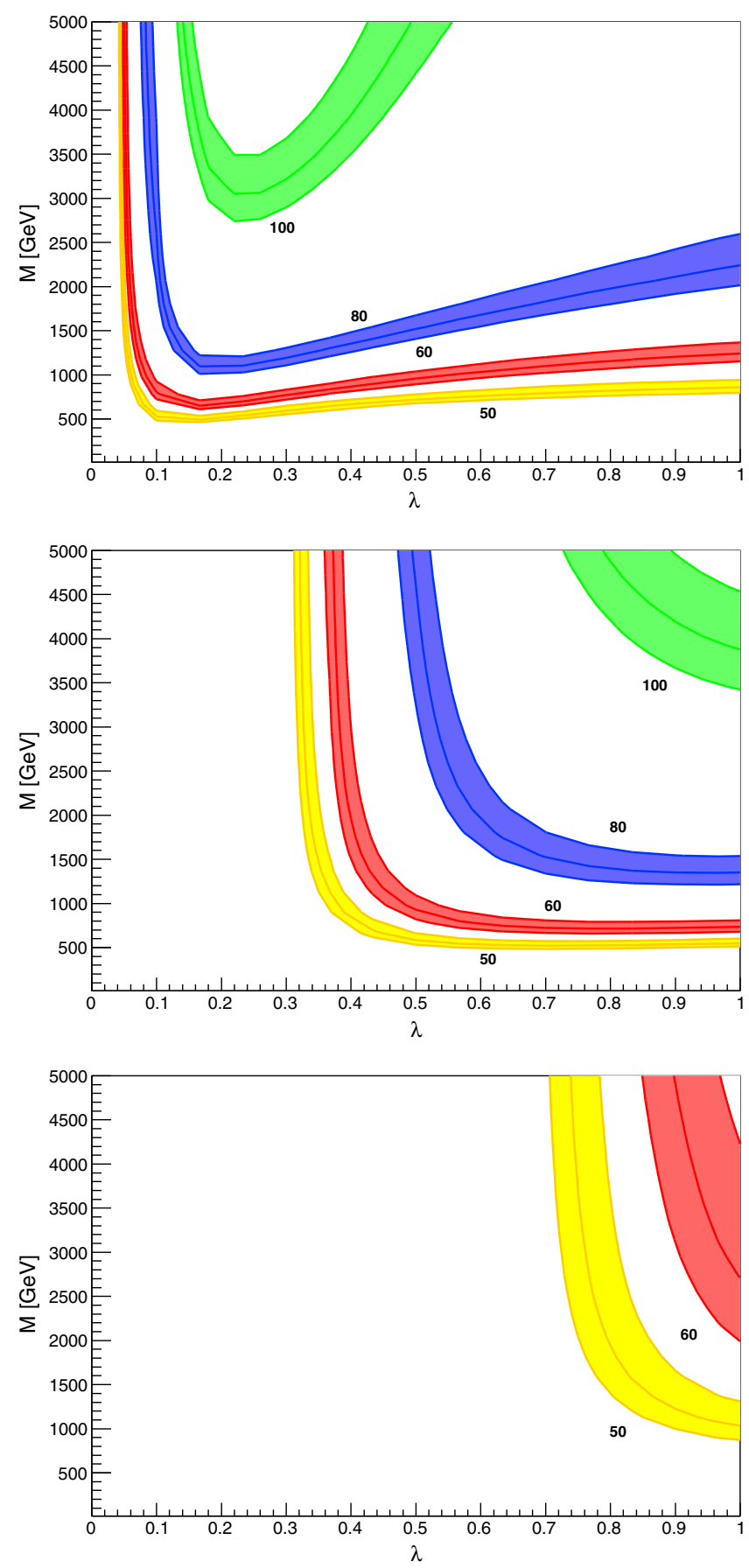

FIG. 4. Limits on the parameter space of our scenario from the ATLAS 8-TeV displaced-vertex search with an integrated luminosity of $20.3 \mathrm{fb}^{-1}$ [10], combining the $\mu \mu$ and $e \mu$ channels. The region to the right and above each colored line is excluded. The neutrino mass scale is fixed to be $m_{\nu} \sim 0.05 \mathrm{eV}$, and the neutrino Yukawa couplings are set to $Y_{\nu}=10^{-7}, 5 \times 10^{-7}$, and $10^{-6}$ in the top, middle, and bottom panels, respectively. The yellow, red, blue, and green lines correspond to the sneutrino mass of 50, 60, 80 and $100 \mathrm{GeV}$, respectively, with the bands representing the uncertainties that come from those in Fig. 13(c) of Ref. [10]. with a lower limit for $\lambda$ of about 0.3 . The case of $Y_{\nu}=10^{-6}$ in the bottom panel is more extreme, and the lower limit on $\lambda$ is now of about 0.7 . Finally, in Fig. 5 we show the same cases as in Fig. 4 but for the neutrino mass scale $0.23 \mathrm{eV}$. To increase the neutrino mass produces an increase in the left sneutrino $\mathrm{VEVs}$, and therefore the $\tilde{\nu}_{\tau}$ decay width into neutrinos is larger. As a consequence, its BR into leptons as
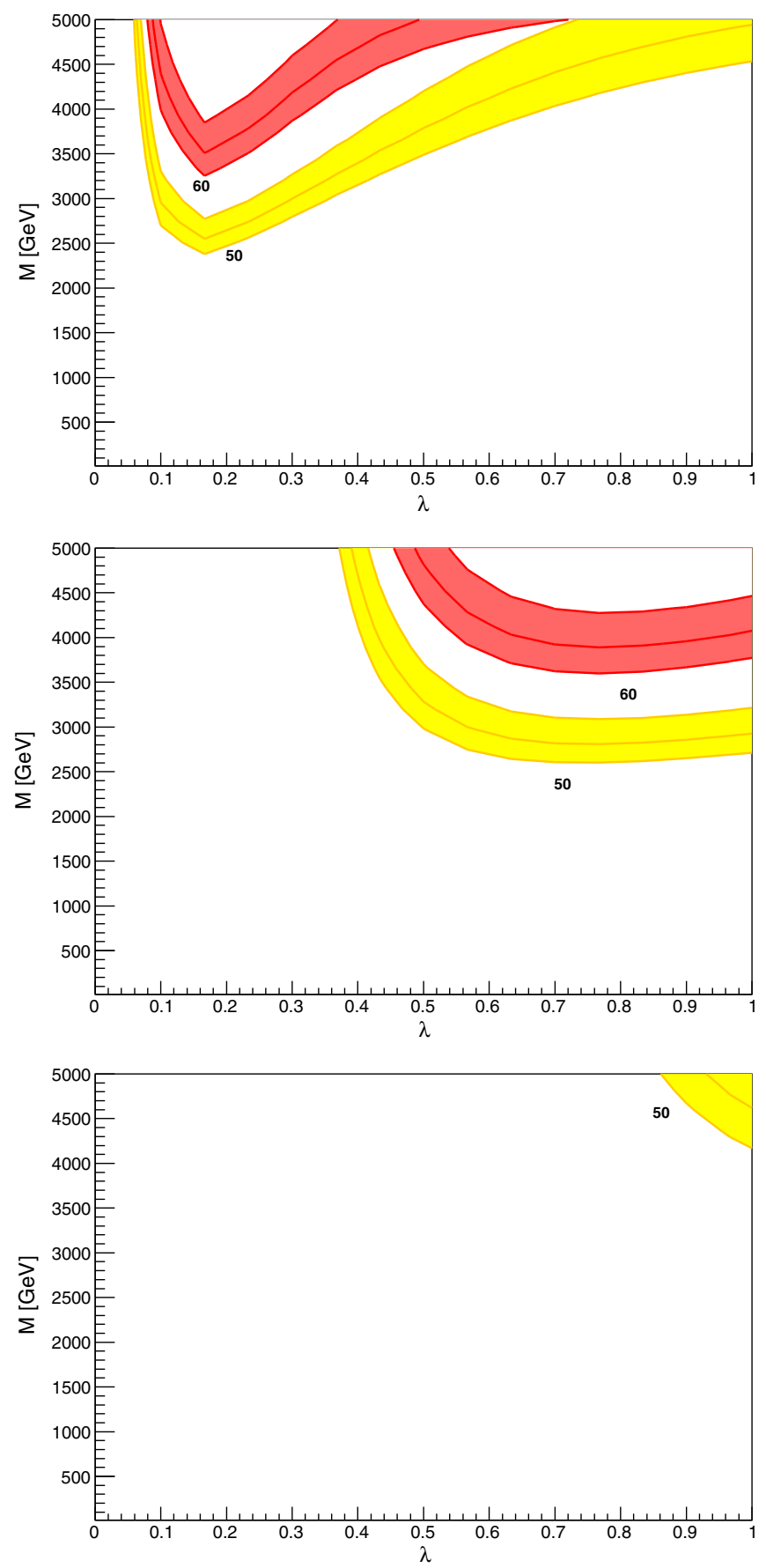

FIG. 5. The same as in Fig. 4 but with the neutrino mass scale fixed to $m_{\nu} \sim 0.23 \mathrm{eV}$. 

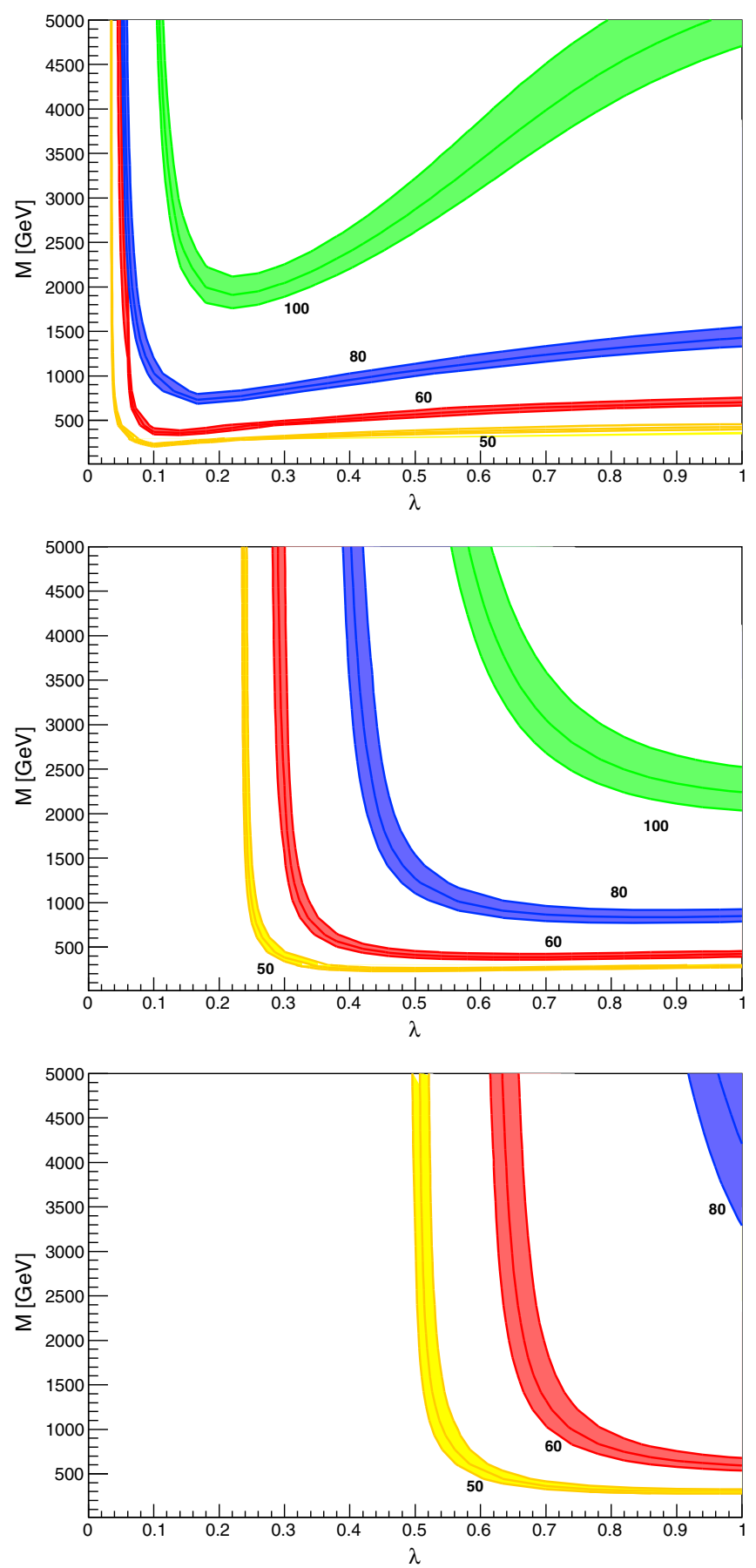

FIG. 6. The same as in Fig. 4 where $m_{\nu} \sim 0.05 \mathrm{eV}$, but considering the optimization of the trigger requirements discussed in the text.

well as its decay length are smaller giving rise to less stringent constraints on the parameter space, as shown in the figure. In particular, there is almost no constraint on the parameter space shown in the bottom panel of Fig. 5.

The potential limits using the optimization of the trigger requirements explained in the previous section, are shown in Figs. 6 and 7. They turn out to be more stringent than the previous ones without optimization. For example, we can
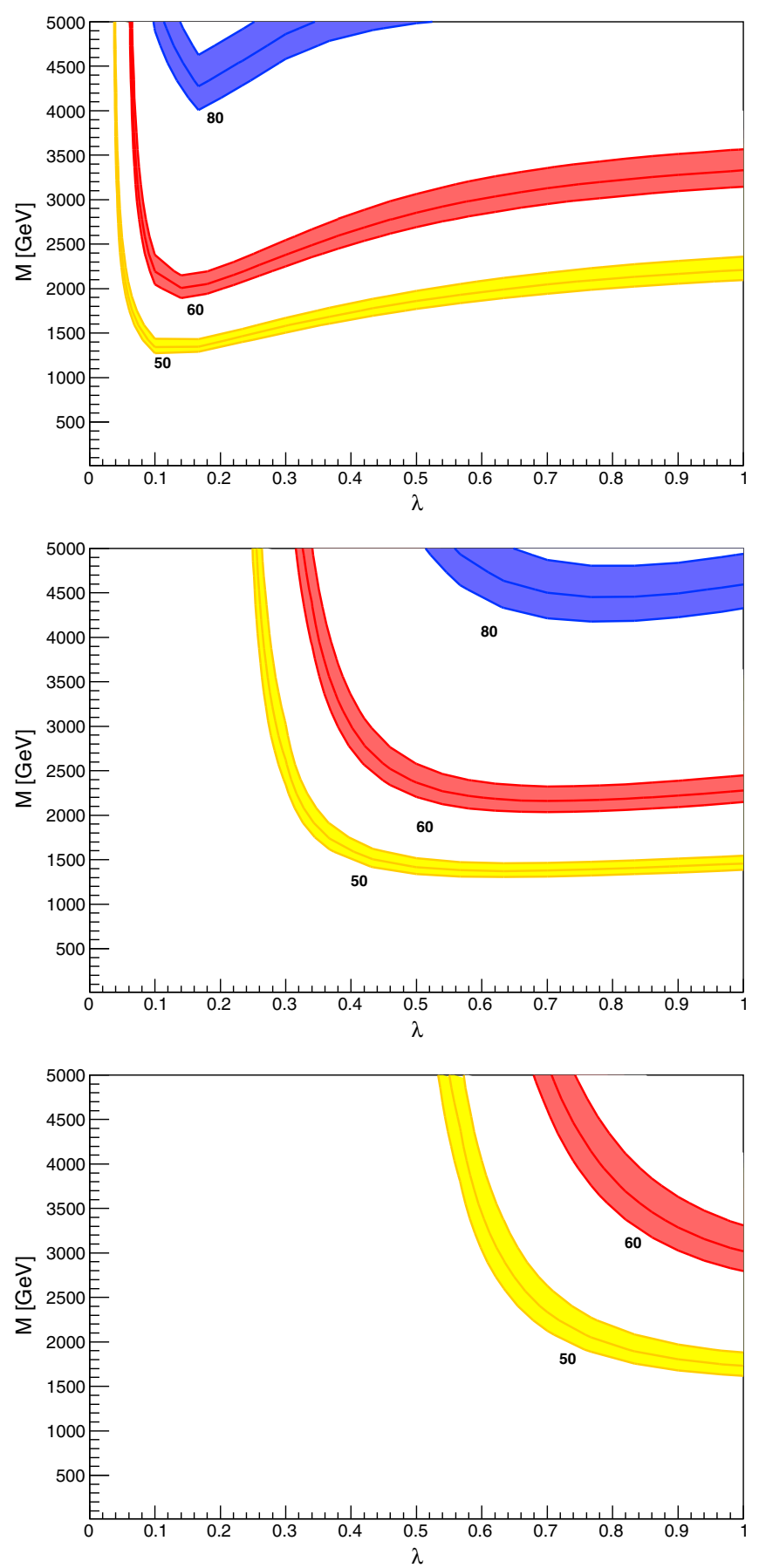

FIG. 7. The same as in Fig. 6 but with the neutrino mass scale fixed to $m_{\nu} \sim 0.23 \mathrm{eV}$.

see in the top panel of Fig. 6 that for $m_{\tilde{\nu}_{\tau}}=50 \mathrm{GeV}$ the upper bound on $M$ is of about 200 (500) $\mathrm{GeV}$ for $\lambda=0.1$ (1), to be compared with the ones of Fig. 4 . Moreover, in the case of $Y_{\nu}=10^{-6}$ and $m_{\nu} \sim 0.23 \mathrm{eV}$ shown in the bottom panel in Fig. 7, now some limits are imposed on light sneutrino masses, which can be compared to the bottom panel of Fig. 5. This result indicates that the trigger optimization discussed above can significantly improve the sensitivity of left sneutrino searches. 

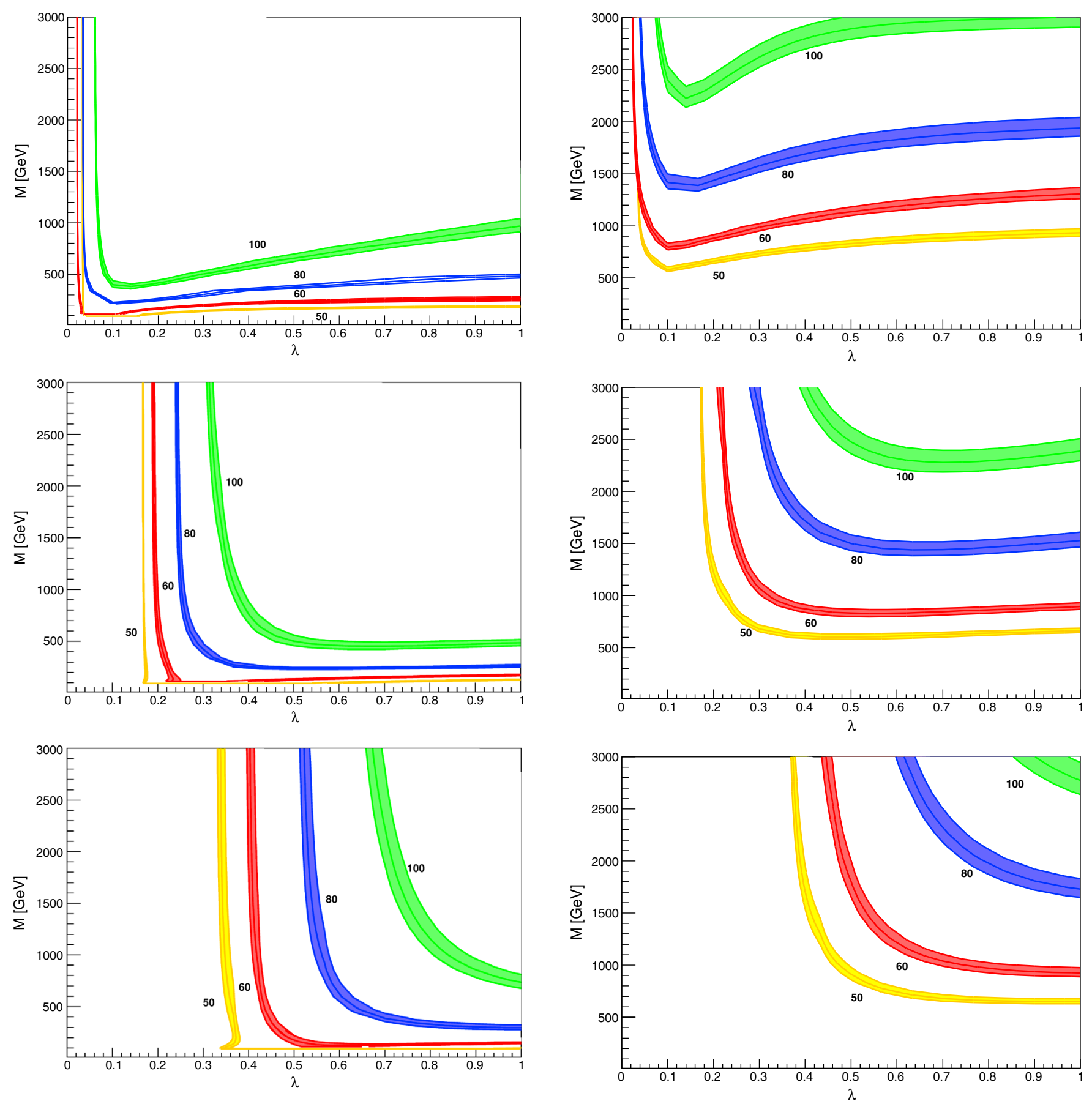

FIG. 8. The same as in Fig. 4 where $m_{\nu} \sim 0.05 \mathrm{eV}$, but analyzing the prospects for the $13-\mathrm{TeV}$ search with an integrated luminosity of $300 \mathrm{fb}^{-1}$, combining the $\mu \mu, e \mu$ and $e e$ channels, and considering also the optimization of the trigger requirements discussed in the text.

The $13 \mathrm{TeV}$ prospects are illustrated in Figs. 8 and 9. Here, we combine the $\mu \mu, e \mu$ and $e e$ channels. As we can see, the constraints on the parameter space of our scenario turn out to be very strong. For example, in the top panel of Fig. 8 and for $\lambda=0.1$, the upper bound on $M$ is as small as about 100, 200, and $350 \mathrm{GeV}$ for $m_{\tilde{\nu}_{\tau}} \approx 50,80$, and

FIG. 9. The same as in Fig. 8 but with the neutrino mass scale fixed to $m_{\nu} \sim 0.23 \mathrm{eV}$.

$100 \mathrm{GeV}$. Such a small $M$ region would be probed in other strategies like ordinary electroweak gaugino searches, which makes it possible to cover a considerable range of the parameter space for the left sneutrino LSP with a mass in the range $45-100 \mathrm{GeV}$. Furthermore, now it is possible to probe a wide range of the parameter space even for the heavier neutrino mass case, $m_{\nu} \sim 0.23 \mathrm{eV}$, as shown in Fig 9. All in all, we conclude that the dilepton 
displaced-vertex searches can be a powerful probe of the $\mu \nu \mathrm{SSM}$ parameter space, especially if we optimize them by making the most of the performance of the inner detectors of the ATLAS and CMS experiments.

\section{CONCLUSIONS AND DISCUSSIONS}

We have analyzed the sensitivity of the displaced dilepton searches at the LHC to a tau left sneutrino LSP with a mass in the range $45-100 \mathrm{GeV}$ in the framework of the $\mu \nu \mathrm{SSM}$. The sneutrino LSP is produced via the $Z$-boson mediated Drell-Yan process or through the $W$ - and $\gamma / Z$ mediated process accompanied with the production and decay of the left stau NLSP. Due to the $R_{p}$ violating term present in the $\mu \nu \mathrm{SSM}$, the left sneutrino LSP becomes metastable and eventually decays into the standard model leptons. Because of the large value of the tau Yukawa coupling, a large fraction of the sneutrino LSP decays into a pair of tau leptons or a tau lepton and a light charged lepton, while the rest decays into a pair of neutrinos. It is found that the decay distance of the left sneutrino tends to be as large as $\gtrsim 1 \mathrm{~mm}$, which thus can be a good target of displaced vertex searches. We have found that the displaced dilepton search channel is most sensitive to the sneutrino LSP, where at least one of the pair-produced left sneutrinos is required to decay into $\tau \tau$ or $\tau \ell$ with the final-state tau leptons decaying leptonically. To evaluate the prospects of this search strategy, we recast the result of the ATLAS 8-TeV dilepton search to obtain the potential limit on the parameter space of our scenario from the 8-TeV LHC data. It is found that even the present data set potentially gives a constraint on the left sneutrino LSP, especially when the Yukawa couplings and mass scale of neutrinos are rather small. We have also discussed an optimization of the trigger requirements exploited in the ATLAS search based on a high level trigger that utilizes the tracker information. It turns out that this optimization can considerably improve the sensitivity of the displaced dilepton search. Moreover, we have estimated the potential limits obtained at the 13$\mathrm{TeV} \mathrm{LHC}$ run and found that a wide range of the parameter space of our scenario can be probed at the LHC run 3.

As mentioned above, we may consider further optimization for the sneutrino LSP search. Given the low background in the dilepton displaced-vertex search, we may relax the condition on the impact parameter of lepton tracks used for the reconstruction of displaced vertices, as well as that on the reconstructed position of displaced vertices. With such a relaxation, it may be possible to detect submillimeter dilepton displaced vertices, which allows us to probe sneutrinos with a shorter lifetime. A further optimization for the trigger requirements is another interesting option to improve the potential of this search. For instance, we may also use the dilepton triggers, which accommodate a lower momentum threshold. Such optimizations highly rely on the detector performance and thus a more dedicated study is required to assess their prospects.

The displaced sneutrino decay signature is useful not only for its discovery but also for the determination of parameters relevant to the sneutrino decay properties. For example, measurement of the lifetime of the sneutrino LSP through the reconstruction of displaced vertices allows us to constrain the parameters in Eq. (2.6), such as $Y_{\nu}, M$, and $\lambda$. In addition, it is in principle possible to measure the mass of the sneutrino LSP since it can decay into visible particles such as $\tau \tau$ and $\tau \ell$; by using hadronically decaying tau leptons, we may fully reconstruct the momenta of the finalstate leptons. Although this may be rather challenging, it is worth investigating this possibility in the future.

In this paper, we focus on the simplest case of the $\mu \nu \mathrm{SSM}$ with one right-handed neutrino superfield. Of course, the metastable left sneutrino can also appear if we introduce three right-handed neutrinos so that they give masses to light neutrinos at tree level. In this case, the sneutrino couplings should be chosen so that the neutrino oscillation data is reproduced, which may have some implications for the sneutrino decay properties. Another interesting possibility is to consider a different LSP, which can be again long-lived due to the small $R_{p}$ violation. In particular, a colored LSP such as the stop LSP may be interesting as its production cross section is quite large at the LHC. Even in this case, we may still probe it by searching for, e.g., multitrack displaced vertices. These subjects will be discussed on another occasion [9].

\section{ACKNOWLEDGMENTS}

N. N. and C. M. thank K. A. Olive and his family for their hospitality at their home, where the present work was initiated, during "Olivefest: Astroparticle Physics Looking Forward." The work of I. L. and C. M. was supported in part by the Spanish Agencia Estatal de Investigación through Grant No. FPA2015-65929-P (MINECO/ FEDER, UE) and IFT Centro de Excelencia Severo Ochoa SEV-2016-0597. The work of D. L. was supported by the Argentinian CONICET, and he also acknowledges the support of the Spanish Grant No. FPA2015-65929-P (MINECO/FEDER, UE). The work of N. N. was supported by the Grant-in-Aid for Scientific Research (No. 17K14270). The work of R. R. was supported in part by Grant No. FPA2014-57816-P, and the Program SEV2014-0398 “Centro de Excelencia Severo Ochoa." I. L., C. M., D. L. and R. R. also acknowledge the support of the MINECO's Consolider-Ingenio 2010 Programme under grant MultiDark CSD2009-00064. 
[1] D. E. López-Fogliani and C. Muñoz, Proposal for a Supersymmetric Standard Model, Phys. Rev. Lett. 97, 041801 (2006).

[2] N. Escudero, D. E. López-Fogliani, C. Muñoz, and R. R. de Austri, Analysis of the parameter space and spectrum of the $\mu \nu$ SSM, J. High Energy Phys. 12 (2008) 099.

[3] P. Ghosh and S. Roy, Neutrino masses and mixing, lightest neutralino decays and a solution to the $\mu$ problem in supersymmetry, J. High Energy Phys. 04 (2009) 069.

[4] A. Bartl, M. Hirsch, A. Vicente, S. Liebler, and W. Porod, LHC phenomenology of the $\mu \nu$ SSM, J. High Energy Phys. 05 (2009) 120.

[5] J. Fidalgo, D. E. López-Fogliani, C. Muñoz, and R. R. de Austri, Neutrino physics and spontaneous $C P$ violation in the $\mu \nu$ SSM, J. High Energy Phys. 08 (2009) 105.

[6] P. Ghosh, P. Dey, B. Mukhopadhyaya, and S. Roy, Radiative contribution to neutrino masses and mixing in $\mu \nu \mathrm{SSM}$, J. High Energy Phys. 05 (2010) 087.

[7] D. E. López-Fogliani and C. Muñoz, On a reinterpretation of the Higgs field in supersymmetry and a proposal for new quarks, Phys. Lett. B 771, 136 (2017).

[8] P. Ghosh, I. Lara, D. E. Lopez-Fogliani, C. Munoz, and R. R. de Austri, Searching for left sneutrino LSP at the LHC, Int. J. Mod. Phys. A 33, 1850110 (2018).

[9] E. Kaptcha, I. Lara, D. E. López-Fogliani, C. Muñoz, N. Nagata, H. Otono et al. (to be published).

[10] G. Aad et al. (ATLAS Collaboration), Search for massive, long-lived particles using multitrack displaced vertices or displaced lepton pairs in pp collisions at $\sqrt{s}=8 \mathrm{TeV}$ with the ATLAS detector, Phys. Rev. D 92, 072004 (2015).

[11] V. Khachatryan et al. (CMS Collaboration), Search for longlived particles that decay into final states containing two electrons or two muons in proton-proton collisions at $\sqrt{s}=8 \mathrm{TeV}$, Phys. Rev. D 91, 052012 (2015).

[12] S. Dawson, E. Eichten, and C. Quigg, Search for supersymmetric particles in hadron-hadron collisions, Phys. Rev. D 31, 1581 (1985).

[13] E. Eichten, I. Hinchliffe, K. D. Lane, and C. Quigg, Super collider physics, Rev. Mod. Phys. 56, 579 (1984).

[14] F. del Aguila and L. Ametller, On the detectability of sleptons at large hadron colliders, Phys. Lett. B 261, 326 (1991).

[15] H. Baer, C.-h. Chen, F. Paige, and X. Tata, Detecting sleptons at hadron colliders and supercolliders, Phys. Rev. D 49, 3283 (1994).

[16] H. Baer, B. W. Harris, and M. H. Reno, Next-to-leading order slepton pair production at hadron colliders, Phys. Rev. D 57, 5871 (1998).

[17] G. Bozzi, B. Fuks, and M. Klasen, Slepton production in polarized hadron collisions, Phys. Lett. B 609, 339 (2005).

[18] P. Abreu et al. (DELPHI Collaboration), Search for supersymmetry with R-parity violating $\mathrm{L} \mathrm{L}$ anti-E couplings at S**(1/2)=183-GeV, Eur. Phys. J. C 13, 591 (2000).

[19] P. Abreu et al. (DELPHI Collaboration), Search for SUSY with R-parity violating LL anti-E couplings at $\mathrm{s}^{* *(1 / 2)}$ =189-GeV, Phys. Lett. B 487, 36 (2000).

[20] P. Achard et al. (L3 Collaboration), Search for R parity violating decays of supersymmetric particles in $e^{+} e^{-}$ collisions at LEP, Phys. Lett. B 524, 65 (2002).

[21] A. Heister et al. (ALEPH Collaboration), Search for supersymmetric particles with $\mathrm{R}$ parity violating decays in $e^{+} e^{-}$collisions at $\sqrt{s}$ up to 209-GeV, Eur. Phys. J. C 31, 1 (2003).

[22] G. Abbiendi et al. (OPAL Collaboration), Search for R parity violating decays of scalar fermions at LEP, Eur. Phys. J. C 33, 149 (2004).

[23] J. Abdallah et al. (DELPHI Collaboration), Search for supersymmetric particles assuming R-parity nonconservation in e+ e- collisions at $\mathrm{s}^{* *}(1 / 2)=192-\mathrm{GeV}$ to $208-\mathrm{GeV}$, Eur. Phys. J. C 36, 1 (2004).

[24] J. Abdallah et al. (DELPHI Collaboration), Photon events with missing energy in e+ e- collisions at $\sqrt{s}=130$ to 209 GeV, Eur. Phys. J. C 38, 395 (2005).

[25] M. Aaboud et al. (ATLAS Collaboration), Search for new phenomena in events with a photon and missing transverse momentum in $p p$ collisions at $\sqrt{s}=13 \mathrm{TeV}$ with the ATLAS detector, J. High Energy Phys. 06 (2016) 059.

[26] M. Aaboud et al. (ATLAS Collaboration), Search for new phenomena in final states with an energetic jet and large missing transverse momentum in $p p$ collisions at $\sqrt{s}=$ 13 TeV using the ATLAS detector, Phys. Rev. D 94, 032005 (2016).

[27] G. Abbiendi et al. (OPAL Collaboration), Search for chargino and neutralino production at $\sqrt{s}=192-209 \mathrm{GeV}$ at LEP, Eur. Phys. J. C 35, 1 (2004).

[28] F. Staub, SARAH, arXiv:0806.0538.

[29] W. Porod, SPheno, a program for calculating supersymmetric spectra, SUSY particle decays and SUSY particle production at e+ e- colliders, Comput. Phys. Commun. 153, 275 (2003).

[30] W. Porod and F. Staub, SPheno 3.1: Extensions including flavor, $C P$ phases and models beyond the MSSM, Comput. Phys. Commun. 183, 2458 (2012).

[31] P. A. R. Ade et al. (Planck Collaboration), Planck 2015 results. XIII. Cosmological parameters, Astron. Astrophys. 594, A13 (2016).

[32] F. P. An et al. (Daya Bay Collaboration), New Measurement of Antineutrino Oscillation with the Full Detector Configuration at Daya Bay, Phys. Rev. Lett. 115, 111802 (2015).

[33] M. Aaboud et al. (ATLAS Collaboration), Search for longlived, massive particles in events with displaced vertices and missing transverse momentum in $\sqrt{s}=13 \mathrm{TeV} p p$ collisions with the ATLAS detector, Phys. Rev. D 97, 052012 (2018).

[34] J. Alwall, R. Frederix, S. Frixione, V. Hirschi, F. Maltoni, O. Mattelaer, H.-S. Shao, T. Stelzer, P. Torrielli, and M. Zaro, The automated computation of tree-level and next-toleading order differential cross sections, and their matching to parton shower simulations, J. High Energy Phys. 07 (2014) 079.

[35] T. Sjostrand, S. Mrenna, and P.Z. Skands, A brief introduction to PYTHIA 8.1, Comput. Phys. Commun. 178, 852 (2008).

[36] B. Fuks, M. Klasen, D. R. Lamprea, and M. Rothering, Revisiting slepton pair production at the Large Hadron Collider, J. High Energy Phys. 01 (2014) 168.

[37] J. de Favereau, C. Delaere, P. Demin, A. Giammanco, V. Lemaitre, A. Mertens, and M. Selvaggi (DELPHES 3 Collaboration), DELPHES 3, A modular framework for 
fast simulation of a generic collider experiment, J. High Energy Phys. 02 (2014) 057.

[38] G. Aad et al. (ATLAS Collaboration), Performance of the ATLAS muon trigger in pp collisions at $\sqrt{s}=8 \mathrm{TeV}$, Eur. Phys. J. C 75, 120 (2015).

[39] G. Aad et al. (ATLAS Collaboration), Performance of the ATLAS trigger system in 2010, Eur. Phys. J. C 72, 1849 (2012).

[40] G. Aad et al. (ATLAS Collaboration), The ATLAS experiment at the CERN Large Hadron Collider, J. Instrum. 3, S08003 (2008).

[41] ATLAS, https://twiki.cern.ch/twiki/pub/AtlasPublic/ EgammaTriggerPublicResults/Plots2014ICHEP_Fig2a .pdf.

[42] M. Aaboud et al. (ATLAS Collaboration), Performance of the ATLAS trigger system in 2015, Eur. Phys. J. C 77, 317 (2017).

[43] ATLAS, https://twiki.cern.ch/twiki/bin/view/AtlasPublic/ MuonTriggerPublicResults\#Plots_for_2017_data_13_TeV.

[44] ATLAS, https://twiki.cern.ch/twiki/bin/view/AtlasPublic/ EgammaTriggerPublicResults\#2017_Data_13_TeV.
[45] Track reconstruction performance of the ATLAS inner detector at $\sqrt{s}=13 \mathrm{TeV}$, Technical Report No. ATLPHYS-PUB-2015-018, CERN, Geneva, 2015.

[46] H. Ito, O. Jinnouchi, T. Moroi, N. Nagata, and H. Otono, Extending the LHC reach for new physics with submillimeter displaced vertices, Phys. Lett. B 771, 568 (2017).

[47] H. Ito, O. Jinnouchi, T. Moroi, N. Nagata, and H. Otono, Searching for metastable particles with submillimeter displaced vertices at hadron colliders, J. High Energy Phys. 06 (2018) 112.

[48] V. Khachatryan et al. (CMS Collaboration), Search for Displaced Supersymmetry in Events with an Electron and a Muon with Large Impact Parameters, Phys. Rev. Lett. 114, 061801 (2015).

[49] V. Khachatryan et al. (CMS Collaboration), Search for R-parity violating supersymmetry with displaced vertices in proton-proton collisions at $\sqrt{s}=8 \mathrm{TeV}$, Phys. Rev. D 95, 012009 (2017).

[50] CMS Collaboration, Search for displaced leptons in the e- $\mu$ channel, Technical Report No. CMS-PAS-EXO-16-022, CERN, Geneva, 2016. 